Abiodun O. Ajibade and Ayuba M. Umar*

\title{
Mixed convection flow in a vertical channel in the presence of wall conduction, variable thermal conductivity and viscosity
}

https://doi.org/10.1515/nleng-2020-0026

Received Sep 24, 2019; accepted Nov 3, 2020.

\begin{abstract}
This study theoretically investigates the effects of variable viscosity, thermal conductivity and wall conduction on a steady mixed convection flow of heat generating/absorbing fluid passes through a vertical channel. One of the channel plates moves with a constant velocity while the other is stationary. The governing flow equations are solved analytically using homotopy perturbation method (HPM). The effects of the thermophysical and hydrodynamics parameters are captured in graphs and tables. It has been observed that, both the velocity and temperature distributions decrease with increase in viscosity and boundary plate thickness near the heated plate while a reverse cases were observed near the cold plate. Increase in thermal conductivity $\varepsilon$ decreases the fluid flow near the heated plate. When the boundary plate thickness is increased, the critical value of Gre to onset the reverse flow increases while increase in thermal conductivity reduces the critical value of Gre. It's also noticed that the skin friction and rate of heat transfer at the heated plate decrease with increase in boundary plate thickness $d$.
\end{abstract}

Keywords: mixed convection, viscous dissipation, vertical channel, boundary plate thickness, mass flux, variable viscosity, variable thermal conductivity

\section{Nomenclature}

$k_{1}$ : Thermal conductivity of plate $p_{1}\left(W m^{-1} s^{-1}\right)$

$k_{2}$ : Thermal conductivity of plate $p_{2}\left(W m^{-1} s^{-1}\right)$

$\mu^{\star}$ : Dimensional viscosity $\left(m^{2} s^{-1}\right)$

$k^{\star}$ : Dimensional thermal conductivity of the fluid

Abiodun 0. Ajibade, Department of Mathematics, Ahmadu Bello University, Zaria, Nigeria, E-mail: aoajibade@abu.edu.ng *Corresponding Author: Ayuba M. Umar, Division of Agricultural Colleges, Ahmadu Bello University, Zaria, Nigeria, E-mail: amumar@abu.edu.ng
$\left(W m^{-1} s^{-1}\right)$

$\lambda$ : Variable viscosity of the fluid $\left(\mathrm{m}^{2} \mathrm{~s}^{-1}\right)$

$\varepsilon$ : Variable thermal conductivity of the fluid $\left(\mathrm{Wm}^{-1} \mathrm{~s}^{-1}\right)$

$u^{\star}$ : Dimensional velocity $\left(m s^{-1}\right)$

$y^{\star}$ : Dimensional distance between the plates $(m)$

$\rho$ : Fluid density $\left(\mathrm{kgm}^{-3}\right)$

$g:$ Acceleration due to gravity $\left(\mathrm{ms}^{-2}\right)$

$\beta$ : Thermal expansion coefficient $\left(K^{-1}\right)$

$T_{f}^{\star}$ : Dimensional temperature of the fluid $(K)$

$c_{p}$ : Specific heat at constant pressure $\left(\mathrm{Kg}^{-1} \mathrm{~K}^{-1}\right)$

$Q_{0}$ : Heat absorption coefficient

$T_{0}$ : Temperature of the cold plate $(K)$

$T_{w}$ : Temperature of the heated plate $(K)$

$T_{p_{1}}^{\star}$ : Dimensional temperature of plate $p_{1}(K)$

$T_{p_{2}}^{\star}$ : Dimensional temperature of plate $p_{2}(K)$

$T_{p_{1}}$ : Temperature of plate $p_{1}(K)$

$T_{p_{2}}$ : Temperature of plate $p_{2}(K)$

$h$ : Channel width $(m)$

$U$ : Velocity of the heated plate $\left(m s^{-1}\right)$

$x$ : Distance along $y$-direction $(m)$

$y$ : Distance along $x$-direction $(m)$

$\frac{k_{0}}{k_{1}}$ : thermal conductivity ratio of fluid and plate $p_{1}$

$\frac{k_{0}}{k_{2}}$ : thermal conductivity ratio of fluid and plate $p_{2}$

$P^{\star}$ : Dimensional pressure $(\mathrm{Pa})$

$d^{*}$ : Dimensional plate thickness $(m)$

$d$ : Plate thickness $(m)$

\section{Introduction}

Heat transfer and fluid flow have been matters of research for many years due to their applications in modern industries. Fluid flows are captured in many mathematical geometries. Such flows could be laminar or turbulent. Fluid dynamics has so many applications in lubrication industries, gas turbine power plant, polymer technology, food processing industries, plasma power plant, thermal and insulating engineering, MHD power generators etc. These fluid flows are driven either by natural, forced or mixed convection. 
Ajay and Feffrey [1] studied combined forced and natural convection heat transfer where they showed that the velocity profile reduces near the channel plate $(y=0)$ with increasing mixed convection while the reverse case was observed at the other plate $(y=1)$. Jha et al. [2] examined mixed convection flow in a vertical tube filled with porous material. They found that the velocity decreases near the inner surface with increasing mixed convection while it increases on the surface of the outer cylinder. Mohamed [3] studied transient mixed convection heat transfer along a vertical stretching surface in the presence of variable viscosity and viscous dissipation. He concluded that the effect of the mixed convection on the velocity and temperature in the steady flow is more prominent than the transient flow. He further discovered that the flow and thermal distributions are enhanced as the strength of the mixed convection increases. Jha et al. [4] studied mixed convection flow in an inclined channel filled with porous material and concluded that the flow is reversal type, since the velocity profile increased by enhancing the value of mixed convection on the lower inclined channel wall while the trend increased with increase in negative value of the mixed convection on the heated wall. Finite element analysis of heat and mass transfer by MHD mixed convection flow of a non-Newtonian power-law nanofluid was investigated by Machal and Naikoti [5]. They concluded that, the velocity profile increases with increase in mixed convection while the reverse case was observed on the fluid temperature. Recently, Jha and Aina [6] studied effect of heat generation/absorption on MHD mixed convection flow in a vertical tube. They concluded that, heat generation increases the rate of heat transfer, while the reverse trend was observed in the case of heat absorption. Entropy generation and irreversibility analysis on a steady mixed convection flow in a vertical porous channel was investigated by Ajibade and Onoja [7]. They revealed that the velocity profile increases with increase in mixed convection while a reverse case was observed for temperature distribution. Jha and Aina [8] analysed the effect of induced magnetic field on MHD mixed convection flow in vertical microchannel.

In lubrication industries, viscous dissipation effects cannot be overlooked since internal energy is generated as a result of fluid particles' interaction which affects the fluid flow and thermal behaviour in a medium. Pantokratoras [9] studied effects of viscous dissipation in natural convection flow along a heated vertical plate. They concluded that the viscous dissipation has a strong effect on velocity profile as it helps the upward and opposes the downward flow. Jha and Ajibade [10] studied effect of viscous dissipation on natural convection flow between vertical parallel plates. They showed that the viscous dissipation heating within the channel increases the fluid temperature above the plate temperature when the Prandtl number of the working fluid is relatively small. Kumar and Sanchayan [11] investigated effects of viscous dissipation on the limiting value of Nusselt numbers for a shear driven flow between two asymmetrically heated plates. They concluded that the temperature profile and the driven temperature difference increase with an increase in viscous dissipation. In a related work, Fransisca et al. [12] examined heat transfer with viscous dissipation in Couette-Poiseuille flow and found that viscous dissipation has significant influence on the fluid temperature. Recently, Kabir et al. [13] investigated effects of viscous dissipation on MHD natural convection flow with heat generation and found that the velocity and temperature profiles increase with increasing viscous dissipation. Mohamed [14] examined mixed convection flow on a micropolar fluid over an unsteady stretching surface in the presence of viscous dissipation. He concluded that the velocity and the temperature profiles increase with increase in Eckert number. Prabhakar [15] examined effects of thermal diffusion and viscous dissipation on an unsteady Magnetohydrodynamics free convection flow past a vertical porous plate in the presence of heat sink. Their work concluded that the velocity and temperature profiles increase with increase in viscous dissipation. RaihanulHaque et al. [16] examined effects of viscous dissipation on natural convection flow over a sphere with temperature dependent thermal conductivity in the presence of heat generation. They found that the velocity and temperature profiles increase with increase in viscous dissipation. All the reviewed articles mentioned above made a common assumption and that is to assume that the boundary plates in each of their investigation is of negligible thickness or rather, heat conduction through the boundary plates plays an insignificant role in the hydrodynamics and thermal behaviour within the channel.

The boundary plate thickness of a material has significant effects on the heat flux and fluid flow through a channel with thick-plate boundaries. This is in the conclusion reached in an earlier investigation of Ajibade and Umar [17] in which it was discovered that the rate of heat transfer from the plate to the fluid decreases with growing thickness in the boundary plates. In the study of effects of wall heat conduction on the reforming process of methane in microreactor, Michael and Dimos [18] concluded that heat transport through the solid wall depends on the crosssectional area of that solid. Hassab et al. [19] analysed effect of axial wall conduction on heat transfer between two parallel plates. Their result showed that, increase in wall thickness serves as thermal resistance to the heat trans- 
fer. Ates et al. [20] analysed a transient conjugated heat transfer on a thick walled pipes and found that more heat penetrated backwardly through the upstream region by axial conduction in the thick wall. Mei et al. [21] examined criteria of axial wall heat conduction under two classical thermal boundary conditions and showed that the temperature gradient number of the thin-wall tube is higher than that of thick-wall tube. The effects of wall conduction on a mixed convection heat transfer in an externally finned pipes were studied by Moukalled et al. [22]. They observed that the axial wall conduction in the pipe wall is found to have strong effect on the flow and thermal fields. Other works that investigated the effect of boundary plates' thickness include the work of Kevin and Barbaros [23].

Convective flow in nature and engineering phenomena requires that viscosity and thermal conductivity of fluids vary with temperature. For instance, the viscosity of dry air at $100^{\circ} \mathrm{C}$ is $21.94 \times 10^{-} 6 \mathrm{~kg} / \mathrm{ms}$ while at $200^{\circ} \mathrm{C}$, it is $26.94 \times 10^{-} 6 \mathrm{~kg} / \mathrm{ms}$. The thermal conductivity of a dry air at $100^{\circ} \mathrm{C}$ is $31.39 \times 10^{-} 3 \mathrm{~W} / \mathrm{mK}$ while at $200^{\circ} \mathrm{C}$, it is $37.95 \times 10^{-} 3 \mathrm{~W} / \mathrm{mK}$ ( Adrian et al. [24]). Tarakaramu et al. [25] investigated the effects of variable thermal conductivity on 3D flow of nanofluid over a stretching sheet using numerical simulation while Dulal and Hiranmony [26] analysed the effects of temperature-dependent viscosity and variable thermal conductivity on mixed convective diffusion flow. They found that velocity profile increases while temperature decreases with increase in mixed convection. Subhas and Mehesha [27] studied heat transfer on a magnetohydrodynamics viscoelastic fluid flow over a stretching sheet with variable thermal conductivity and radiation and found that the variable thermal conductivity has an influence in enhancing the temperature and fluid flow. The works of Rahman et al. [28] and Naseem [29] have shown that the velocity and temperature profiles increase with increase in thermal conductivity. Vajravelu et al. [30], Singh and Shweta [31] and Isaac and Auselm [32] showed that, velocity distributions decrease with increase in viscosity while the temperature profiles increase with increase in variable viscosity. MHD effects on heat transfer over a stretching sheet embedded on a porous medium with variable viscosity, viscous dissipation and heat source/sink were studied by Hunegnaw and Naikoti [33]. Uwanta and Murtala [34] studied heat and mass transfer flow past an infinite vertical plate with variable thermal conductivity, heat source and chemical reaction. They concluded that, velocity and temperature profiles increase with increase in thermal conductivity. Sumayya et al. [35] investigated efficiency analysis of a straight fin with variable heat transfer and thermal conductivity. They con- cluded that, temperature profile increases with increase in thermal conductivity.

Devi and Prakash [36] examined temperature-dependent viscosity and thermal conductivity effects on hydromagnetic flow over a slendering stretching sheet. They concluded that, increase in the viscosity decreases the velocity profile. The work of Animasaun [37] concluded that fluid velocity decreases while temperature increases with increases due to increasing viscosity. The works of Gopal and Jadav [38], Jadav and Hazarika [39], Gopal and Bandita [40], Bandita [41] and Kareem and Salawu [42] have shown that the velocity profiles decrease with increasing viscosity while the temperature profiles increase with increase in viscosity. Recently, Sreenivasulu et al. [43] studied variable thermal conductivity influence on hydromagnetic flow past a stretching cylinder in a thermally stratified medium with heat source/sink. They concluded that the velocity profile increases with increasing mixed convection and the temperature profile increases with increase in thermal conductivity. Muthtamilselvan et al. [44] investigated an internal heat generation of a dusty fluid passes through porous media of a stretching sheet using Range-Kutta fourth order method.

The aim of this work is to carry out a theoretical investigation on the effects of viscous dissipation, wall conduction, boundary plate thickness, variable viscosity and thermal conductivity on a steady mixed convection flow. Due to the wide application of viscous dissipation, conduction at the channel wall, boundary plate thickness in lubrication industries, cooling of nuclear reactors, food processing industries, cooling of electric appliances, plasma physics, aerodynamics etc., it is inappropriate to neglect these important parameters in natural and forced convection as results obtained with constant viscosity and thermal conductivity as well as neglecting the plate thickness and international heat generation as a result of fluidparticle interactions are either under-determined or overdetermined as the case may be. Therefore, it is important to investigate the role of boundary plate thickness, wall conduction, viscous dissipation, variable viscosity and thermal conductivity on mixed convection flow of viscous incompressible heat generating/absorbing fluid.

Obtaining a closed form solution of this model is a very difficult task due to the coupling and nonlinearity nature of the governing equations. Various solution methods have been derived for such problems ranging from numerical solutions (Doh et al. [45], Kairi et al. [46], Kumar et al. [47], Rostami et al. [48], Hosseinzadeh et al. [49], Mukundan and Awasthi [50] etc.), perturbation methods and several other approximate solution techniques (Travis and Erturk [51], Singh et al. [52] etc.), thus, this problem has adopted 
the Homotopy Perturbation Method to carry out its investigations.

\section{Mathematical analysis}

We consider a two-dimensional steady mixed flow of an incompressible viscous fluid passes through vertical parallel plates of some thickness $d^{\star}$. One of the plates moves in the direction of the fluid flow while the other is stationary (see figure 1). The plate at $y^{\star}=-d^{\star}$ which moves uniformly with velocity $U$ is heated to temperature $T_{w}$ while the other at $y^{\star}=h+d^{\star}$ is kept at ambient temperature $T_{0}$. In addition, an external pressure is applied to the flow which gave rise to mixed convection and also the presence of viscous dissipation was considered. The flow is assumed to be along $x^{\star}$ direction and $y^{*}$ is normal to the plates. Internal energy generation is considered as a result of fluid particles interaction and the thermophysical and hydrodynamics properties are assumed to be constant except for viscosity and thermal conductivity that both vary with temperature. The physical model describing the situation, following the works of (Ajibade and Umar [17], Ajibade and Umar [53]) is given as:

$$
\begin{aligned}
\frac{1}{\rho}\left(\mu^{\star} \frac{d^{2} u^{\star}}{d y^{\star 2}}\right)+g \beta\left(T_{f}^{\star}-T_{0}\right)-\frac{1}{\rho} \frac{d P^{\star}}{d x^{\star}} & =0 \\
\frac{k_{1}}{\rho c_{p}} \frac{d^{2} T_{p_{1}}^{\star}}{d y^{\star 2}} & =0 \\
\frac{1}{\rho c_{p}}\left(k^{\star} \frac{d^{2} T_{f}^{\star}}{d y^{\star 2}}\right)-\frac{Q_{0}}{\rho c_{p}}\left(T_{f}^{\star}-T_{0}\right)+\frac{\mu^{\star}}{\rho c_{p}}\left(\frac{d u^{\star}}{d y^{\star}}\right)^{2} & =0 \\
\frac{k_{2}}{\rho c_{p}} \frac{d^{2} T_{p_{2}}^{\star}}{d y^{\star 2}} & =0
\end{aligned}
$$

The first, second and third terms of the momentum equation (1) are the viscosity, thermal buoyancy, and pressure gradient effects while the first, second and third term of the energy equation (3) of the fluid are the thermal conductivity, heat generation/absorption and viscous dissipation effects respectively. Equations (2) and (4) are the energy equations of the bounding slabs $p_{1}$ and $p_{2}$ respectively.

Since the flow is steady, we assume that the appropriate boundary conditions of the model are:

$$
\begin{array}{rlrlrl}
u^{\star}=U & \text { at } & y^{\star} & =0, \\
u^{\star}=0 & \text { at } & y^{\star} & =h, \\
T_{p_{1}}^{\star}=T_{w} & \text { at } & y^{\star} & =-d^{\star}, \\
T_{p_{1}}^{\star}=T_{f}^{\star} & \text { at } & y^{\star} & =0, \\
k_{1} \frac{d T_{p_{1}}^{\star}}{d y^{\star}}=k^{\star} & \frac{d T_{f}^{\star}}{d y^{*}} & \text { at } & y^{\star} & =0,
\end{array}
$$

$$
\begin{aligned}
T_{p_{2}}^{\star}=T_{f}^{\star} \quad \text { at } \quad y^{\star} & =h, \\
k_{2} \frac{d T_{p_{2}}^{\star}}{d y^{\star}}=k^{\star} \frac{d T_{f}^{\star}}{d y^{\star}} \quad \text { at } \quad y^{\star} & =h, \\
T_{p_{2}}^{\star}=T_{0} \quad \text { at } & y^{\star}=h+d^{\star} .
\end{aligned}
$$

The viscosity of the fluid and its thermal conductivity are both assumed to vary linearly with temperature so that

$$
\begin{aligned}
& k^{\star}=k_{0}\left[1-b\left(T_{f}^{\star}-T_{0}\right)\right] \\
& \mu^{\star}=\mu_{0}\left[1-a\left(T_{f}^{\star}-T_{0}\right)\right]
\end{aligned}
$$

where $\mu_{0}$ and $k_{0}$ represent the viscosity and thermal conductivity of fluid temperature $T_{0}, a$ and $b$ are the thermal factors that control the change in viscosity and thermal conductivity respectively with increase in temperature.

In order to harmonize the various dimensions that are involved in the model, the velocity has been scaled up with $U$, the velocity of the moving plate while the horizontal distances; $y^{*}$ and $d^{*}$ were scaled up with $h$. All other dimensionless parameters are as described in eqn (6):

$$
\begin{array}{r}
u=\frac{u^{\star}}{U}, \quad y=\frac{y^{\star}}{h}, \quad T_{f}=\frac{T_{f}^{\star}-T_{0}}{T_{w}-T_{0}}, \quad T_{p_{1}}=\frac{T_{p_{1}}^{\star}-T_{0}}{T_{w}-T_{0}}, \\
T_{p_{2}}=\frac{T_{p_{2}}^{\star}-T_{0}}{T_{w}-T_{0}}, \quad d=\frac{d^{\star}}{h}, \quad P=\frac{P^{\star}}{\rho U^{2}}, \quad x=\frac{x^{\star} v_{0}}{U h^{2}}
\end{array}
$$

Using the dimensionless quantities (6), the momentum and energy Eqs.(1) - (4) are presented as:

$$
\begin{aligned}
& \left(1-\lambda T_{f}\right) \frac{d^{2} u}{d y^{2}}-\lambda \frac{d T_{f}}{d y} \frac{d u}{d y}+G r e T_{f}-\frac{d P}{d x}=0 \\
& \frac{d^{2} T_{p_{1}}}{d y^{2}}=0 \\
& \left(1-\varepsilon T_{f}\right) \frac{d^{2} T_{f}}{d y^{2}}-\varepsilon\left(\frac{d T_{f}}{d y}\right)^{2}-S\left(1-\varepsilon T_{f}\right) T_{f} \\
& +B r\left(1-\varepsilon T_{f}\right)\left(\frac{d u}{d y}\right)^{2}=0 \\
& \frac{d^{2} T_{p_{2}}}{d y^{2}}=0
\end{aligned}
$$

subject to the boundary conditions:

$$
\begin{aligned}
& u=1 \quad \text { at } \quad y=0 \text {, } \\
& u=0 \quad \text { at } y=1 \text {, } \\
& T_{p_{1}}=1 \quad \text { at } \quad y=-d, \\
& T_{p_{1}}=T_{f} \quad \text { at } \quad y=0 \text {, } \\
& \frac{d T_{p_{1}}}{d y}=\left(1-\varepsilon T_{f}\right) \frac{d T_{f}}{d y} \quad \text { at } \quad y=0 \text {, } \\
& T_{p_{2}}=T_{f} \quad \text { at } \quad y=1 \text {, } \\
& \frac{d T_{p_{2}}}{d y}=\left(1-\varepsilon T_{f}\right) \frac{d T_{f}}{d y} \quad \text { at } \quad y=1 \text {, }
\end{aligned}
$$




$$
T_{p_{2}}=T_{0} \quad \text { at } \quad y=1+d,
$$

where

$$
\begin{array}{r}
S=\frac{Q_{0} h^{2}}{k}, \quad E c=\frac{U^{2}}{c_{p}\left(T_{w}-T_{0}\right)}, \operatorname{Pr}=\frac{v}{\alpha}, \quad E c P r=B r, \\
\frac{k_{0}}{k_{1}}=\frac{k_{0}}{k_{2}}=1 G r e=\frac{G r}{R e}=\frac{g \beta\left(T_{w}-T_{0}\right) h^{3}}{v^{2}} \cdot \frac{v}{U h}, \\
\lambda=a\left(T_{w}-T_{0}\right), \quad \varepsilon=b\left(T_{w}-T_{0}\right) .
\end{array}
$$

The parameters of interest that appeared in the dimensionless eqn (10) are; heat generation/absorption, $(S)$, viscous dissipation (Eckert number, Ec), Prandtl number (Pr), Grashof number and Reynold number $\left(\frac{G r}{R e}\right)$, viscosity and conductivity factor parameters $(\lambda)$ and $(\varepsilon)$ respectively. All other physical parameters are defined in nomenclature.

\section{Method of solution}

Several methods ranging from numerical, perturbations and many other approximate solution techniques are used to solve linear, nonlinear and coupled (partial or ordinary) differential equations. Homotopy Perturbation Method (HPM) is one of the easiest approximate solution since the first two iterations are of accuracy than the traditional perturbation techniques and it doesn't require only a small parameter. The method was first initiated by He [54] where he presented the method by considering a nonlinear differential equation

$$
A(u)-f(r)=0, \quad r \in \Omega
$$

subject to the boundary conditions

$$
B\left(u, \frac{\partial u}{\partial n}\right)=0, \quad r \in \Gamma,
$$

where $A$ is the general differential operator, $B$ is a boundary operator, $f(r)$ is a known analytic function, $\Gamma$ is the boundary of the domain $\Omega$. The operator $A$ can be divided into two parts, namely, $L$ and $N$, where $L$ is the linear and $N$ nonlinear parts. Therefore, the nonlinear differential equation can be transformed as:

$$
L(u)+N(u)-f(r)=0 .
$$

Now the convex homotopy can be constructed from the nonlinear differential equation and its boundary condition as:

$v(r, p): \Omega \times[0,1] \rightarrow \Re$ which satisfies

$$
H(v, p)=(1-p)\left[L(v)-L\left(u_{0}\right)\right]+p[A(v)-f(r)]=0
$$

or

$$
H(v, p)=L(v)-L\left(u_{0}\right)+p L\left(u_{0}\right)+p[N(v)-f(r)]=0,
$$

where $p \in[0,1]$ is an embedding parameter, $u_{0}$ is the initial approximation which satisfies the boundary conditions. Therefore, we have

$$
\begin{array}{r}
H(v, 0)=L(v)-L\left(u_{0}\right)=0 \\
H(v, 1)=A(v)-f(r)=0
\end{array}
$$

Now the solution of the nonlinear differential equation is expressed as:

$$
v=v_{0}+p v_{1}+p^{2} v_{2}+p^{3} v_{3}+\ldots
$$

setting $p=1$, the approximate solution can be written as

$$
v=v_{0}+v_{1}+v_{2}+v_{3}+\ldots
$$

Therefore the convex homotopy of momentum and energy equations are expressed as:

$$
\begin{aligned}
& H(u, p)=(1-p)\left[\frac{d^{2} u}{d y^{2}}-\frac{d^{2} v_{0}}{d y^{2}}\right]+p\left[\frac{d^{2} u}{d y^{2}}+G r e T_{f}\right. \\
& \left.-\lambda \frac{d u}{d y} \frac{d T_{f}}{d y}-\lambda T_{f} \frac{d^{2} u}{d y^{2}}-\frac{d P}{d x}\right]=0 .
\end{aligned}
$$

Since the zeroth order equations are linear and can be solved exactly with the boundary conditions, then initial approximation $v_{0}$, is not required. Therefore we have

$$
\frac{d^{2} u}{d y^{2}}=p\left[\frac{d P}{d x}-G r e T_{f}+\lambda \frac{d u}{d y} \frac{d T_{f}}{d y}+\lambda T_{f} \frac{d^{2} u}{d y^{2}}\right]
$$

such that

$$
\begin{array}{r}
u=u_{0}+p u_{1}+p^{2} u_{2}+p^{3} u_{3}+\ldots, \\
T_{f}=T_{f_{0}}+p T_{f_{1}}+p^{2} T_{f_{2}}+p^{3} T_{f_{3}}+\ldots, \\
T_{p_{1}=}=T_{p_{1_{0}}}+p T_{p_{1_{1}}}+p^{2} T_{p_{1_{2}}}+p^{3} T_{p_{1_{3}}}+\ldots, \\
T_{p_{2}=}=T_{p_{2_{0}}}+p T_{p_{2_{1}}}+p^{2} T_{p_{2_{2}}}+p^{3} T_{p_{2_{3}}}+\ldots,
\end{array}
$$

Substituting eqn(15) into eqn(14) and comparing the coefficients of $p^{0}, p^{1}, p^{2}, p^{3}, \ldots$, we have

$$
\begin{aligned}
& p^{0}: \frac{d^{2} u_{0}}{d y^{2}}=0 \\
& p^{1}: \frac{d^{2} u_{1}}{d y^{2}}=\frac{d P}{d x}-\operatorname{GreT}_{f_{0}}+\lambda \frac{d u_{0}}{d y} \frac{d T_{f_{0}}}{d y}+\lambda T_{f_{0}} \frac{d^{2} u_{0}}{d y^{2}} \\
& p^{2}: \frac{d^{2} u_{2}}{d y^{2}}=-\operatorname{GreT}_{f_{1}}+\lambda \frac{d u_{0}}{d y} \frac{d T_{f_{1}}}{d y}+\lambda \frac{d u_{1}}{d y} \frac{d T_{f_{0}}}{d y} \\
& +\lambda T_{f_{0}} \frac{d^{2} u_{1}}{d y^{2}}+\lambda T_{f_{1}} \frac{d^{2} u_{0}}{d y^{2}}
\end{aligned}
$$


similarly, eqns (8),(9) and (10) can be transformed as

$$
\begin{aligned}
& p^{0}: \frac{d^{2} T_{p_{1_{0}}}}{d y^{2}}=0, \\
& p^{1}: \frac{d^{2} T_{p_{1}}}{d y^{2}}=0, \\
& p^{2}: \frac{d^{2} T_{p_{1_{2}}}}{d y^{2}}=0,
\end{aligned}
$$

$$
\begin{gathered}
p^{1}: \frac{d^{2} T_{f_{1}}}{d y^{2}}=\varepsilon T_{f_{0}} \frac{d^{2} T_{f_{0}}}{d y^{2}}+\varepsilon\left(\frac{d T_{f_{0}}}{d y}\right)^{2}-B r\left(\frac{d u_{0}}{d y}\right)^{2}+B r \varepsilon T_{f_{0}}\left(\frac{d u_{0}}{d y}\right)^{2}+S T_{f_{0}}-S \varepsilon T_{f_{0}}^{2} \\
p^{2}: \frac{d^{2} T_{f_{2}}}{d y^{2}}=\varepsilon T_{f_{0}} \frac{d^{2} T_{f_{1}}}{d y^{2}}+\varepsilon T_{f_{1}} \frac{d^{2} T_{f_{0}}}{d y^{2}}+2 \varepsilon \frac{d T_{f_{0}}}{d y} \frac{d T_{f_{1}}}{d y}-2 B r \frac{d u_{0}}{d y} \frac{d u_{1}}{d y}+2 B r \varepsilon T_{f_{0}} \frac{d u_{0}}{d y} \frac{d u_{1}}{d y} \\
+B r \varepsilon T_{f_{1}}\left(\frac{d u_{0}}{d y}\right)^{2}+S T_{f_{1}}-2 S \varepsilon T_{f_{0}} T_{f_{1}}
\end{gathered}
$$

$$
\begin{aligned}
& p^{0}: \frac{d^{2} T_{p_{2_{0}}}}{d y^{2}}=0, \\
& p^{1}: \frac{d^{2} T_{p_{2_{1}}}}{d y^{2}}=0, \\
& p^{2}: \frac{d^{2} T_{p_{2}}}{d y^{2}}=0,
\end{aligned}
$$

and the boundary conditions are transformed as

$$
\begin{aligned}
& u_{0}=1, u_{1}=u_{2}=u_{3}=\ldots=0 \quad \text { at } y=0 \text {, } \\
& u_{0}=u_{1}=u_{2}=u_{3}=\ldots=0 \text { at } y=1 \text {, } \\
& T_{p_{1_{0}}}=1, \quad T_{p_{1_{1}}}=T_{p_{1_{2}}}=\ldots=0 \text { at } y=-d \text {, } \\
& T_{p_{1_{0}}}=T_{f_{0}}, \quad T_{p_{1_{1}}}=T_{f_{1}}, \quad T_{p_{1_{2}}}=T_{f_{2}}, \quad T_{p_{1_{3}}}=T_{f_{3}}, \ldots \text { at } \quad y=0 \text {, } \\
& \frac{d T_{p_{1_{0}}}}{d y}=\frac{d T_{f_{0}}}{d y}, \quad \frac{d T_{p_{1_{1}}}}{d y}-\frac{d T_{f_{1}}}{d y}=-\varepsilon T_{f_{0}} \frac{d T_{f_{0}}}{d y}, \\
& \frac{d T_{p_{1_{2}}}}{d y}-\frac{d T_{f_{2}}}{d y}=-\varepsilon\left[T_{f_{0}} \frac{d T_{f_{1}}}{d y}+T_{f_{1}} \frac{d T_{f_{0}}}{d y}\right], \ldots \quad \text { at } \quad y=0, \\
& T_{p_{2_{0}}}=T_{f_{0}}, \quad T_{p_{2_{1}}}=T_{f_{1}}, \quad T_{p_{2_{2}}}=T_{f_{2}}, \quad T_{p_{2_{3}}}=T_{f_{3}}, \ldots \text { at } y=1 \text {, } \\
& \frac{d T_{p_{2_{0}}}}{d y}=\frac{d T_{f_{0}}}{d y}, \quad \frac{d T_{p_{2_{1}}}}{d y}-\frac{d T_{f_{1}}}{d y}=-\varepsilon T_{f_{0}} \frac{d T_{f_{0}}}{d y} \\
& \frac{d T_{p_{22}}}{d y}-\frac{d T_{f_{2}}}{d y}=-\varepsilon\left[T_{f_{0}} \frac{d T_{f_{1}}}{d y}+T_{f_{1}} \frac{d T_{f_{0}}}{d y}\right], \ldots \quad \text { at } \quad y=1 \text {, } \\
& T_{p_{2_{0}}}=T_{p_{2_{1}}}=T_{p_{2_{2}}}=\ldots=0 \text { at } y=1+d \text {. }
\end{aligned}
$$


Solving from the above eqns(16), (19), (22) and (25) and applying the boundary conditions (28), we have

$$
\begin{gathered}
u_{0}=A_{0} y+A_{1}, \\
T_{p_{1_{0}}}=A_{2} y+A_{3}, \\
T_{f_{0}}=A_{4} y+A_{5}, \\
T_{p_{2_{0}}}=A_{6} y+A_{7}, \\
A_{0}=-1, \quad A_{1}=1, \quad A_{2}=A_{4}=A_{6}=-\frac{1}{(1+2 d)}, \quad A_{3}=A_{5}=A_{7}=1+A_{2} d .
\end{gathered}
$$

Solving eqns(17), (20), (23) and (26) and applying the boundary conditions (28) we have

$$
\begin{gathered}
u_{1}=\frac{y^{2}}{2} \frac{d P}{d x}-G r e\left[A_{4} \frac{y^{3}}{6}+A_{5} \frac{y^{2}}{2}\right]-\lambda A_{4} \frac{y^{2}}{2}+A_{8} y+A_{9}, \\
T_{p_{1}}=A_{10} y+A_{11}, \\
T_{f_{1}}=\varepsilon A_{4}^{2} \frac{y^{2}}{2}-B r \frac{y^{2}}{2}+B r \varepsilon\left[A_{4} \frac{y^{3}}{6}+A_{5} \frac{y^{2}}{2}\right]+S\left[A_{4} \frac{y^{3}}{6}+A_{5} \frac{y^{2}}{2}\right] \\
-S \varepsilon\left[A_{4}^{2} \frac{y^{4}}{12}+A_{4} A_{5} \frac{y^{3}}{3}+A_{5}^{2} \frac{y^{2}}{2}\right]+A_{12} y+A_{13}, \\
T_{p_{21}}=A_{14} y+A_{15}, \\
A_{12}=\frac{A_{9}}{2(1+2 d)}-\frac{\varepsilon A_{4}^{2}}{2(1+2 d)}-\frac{B r \varepsilon}{(1+2 d)}\left[\frac{A_{4}}{6}+\frac{A_{5}}{2}\right]-\frac{S}{(1+2 d)}\left[\frac{A_{4}}{6}+\frac{A_{5}}{2}\right] \\
+\frac{S \varepsilon}{(1+2 d)}\left[\frac{A_{4}^{2}}{12}+\frac{A_{4} A_{5}}{3}+\frac{A_{5}^{2}}{2}\right]+\frac{B r d}{(1+2 d)}-\frac{B r \varepsilon d}{(1+2 d)}\left[\frac{A_{4}}{2}+A_{5}\right] \\
-\frac{S d}{(1+2 d)}\left[\frac{A_{4}}{2}+A_{5}\right]+\frac{S \varepsilon d}{(1+2 d)}\left[\frac{A_{4}^{2}}{3}+A_{4} A_{5}+A_{5}^{2}\right]+\frac{2 \varepsilon A_{4} A_{5} d}{(1+2 d)}, \\
A_{10}=A_{12}-\varepsilon A_{4} A_{5}, \quad A_{11}=A_{13}=A_{10} d, \\
A_{14}=A_{10}-B r+B r \varepsilon\left[\frac{A_{4}}{2}+A_{5}\right]+S\left[\frac{A_{4}}{2}+A_{5}\right]-S \varepsilon\left[\frac{A_{4}^{2}}{3}+A_{4} A_{5}+A_{5}^{2}\right] .
\end{gathered}
$$

Solving eqns(18), (21), (24) and (27) and applying the conditions (28), we have

$$
\begin{aligned}
u_{2}= & -G r e \varepsilon A_{4}^{2} \frac{y^{4}}{24}+G r e B r \frac{y^{4}}{24}-G r e B r \varepsilon\left[A_{4} \frac{y^{5}}{120}+A_{5} \frac{y^{4}}{24}\right]-G r e S\left[A_{4} \frac{y^{5}}{120}+A_{5} \frac{y^{4}}{24}\right] \\
& +\operatorname{Gre\lambda S}\left[A_{4}^{2} \frac{y^{6}}{360}+A_{4} A_{5} \frac{y^{5}}{60}+A_{5}^{2} \frac{y^{4}}{24}\right]-G r e A_{12} \frac{y^{3}}{6}-G r e A_{13} \frac{y^{2}}{3}-\lambda \varepsilon A_{4}^{2} \frac{y^{3}}{6}+B r \lambda \frac{y^{3}}{6} \\
& -\operatorname{Br} \lambda \varepsilon\left[A_{4} \frac{y^{4}}{24}+A_{5} \frac{y^{3}}{6}\right]-S \lambda\left[A_{4} \frac{y^{4}}{24}+A_{5} \frac{y^{3}}{6}\right]+S \lambda \varepsilon\left[A_{4}^{2} \frac{y^{5}}{60}+A_{4} A_{5} \frac{y^{4}}{12}+A_{5}^{2} \frac{y^{3}}{6}\right] \\
& -\lambda A_{12} \frac{y^{2}}{2}+\lambda A_{4} \frac{d P}{d x} \frac{y^{3}}{6}-G r e \lambda\left[A_{4}^{2} \frac{y^{4}}{24}+A_{4} A_{5} \frac{y^{3}}{6}\right]-\lambda^{2} A_{4}^{2} \frac{y^{3}}{6}+\lambda A_{4} A_{8} \frac{y^{2}}{2} \\
& +\lambda \frac{d P}{d x}\left[A_{4} \frac{y^{3}}{6}+A_{5} \frac{y^{2}}{2}\right]-G r e \lambda\left[A_{4}^{2} \frac{y^{4}}{12}+A_{4} A_{5} \frac{y^{3}}{3}+A_{5}^{2} \frac{y^{2}}{2}\right]-\lambda^{2}\left[A_{4}^{2} \frac{y^{3}}{6}+A_{4} A_{5} \frac{y^{2}}{2}\right] \\
& A_{16} y+A_{17},
\end{aligned}
$$




$$
T_{p_{1_{2}}}=A_{18} y+A_{19},
$$

$$
\begin{aligned}
& T_{f_{2}}=\varepsilon^{2}\left[A_{4}^{3} \frac{y^{3}}{6}+A_{4}^{2} A_{5} \frac{y^{2}}{2}\right]-B r \varepsilon\left[A_{4} \frac{y^{3}}{6}+A_{5} \frac{y^{2}}{2}\right]+B r \varepsilon^{2}\left[A_{4}^{2} \frac{y^{4}}{12}+A_{4} A_{5} \frac{y^{3}}{3}+A_{5}^{2} \frac{y^{2}}{2}\right] \\
& +S \varepsilon\left[A_{4}^{2} \frac{y^{4}}{12}+A_{4} A_{5} \frac{y^{3}}{3}+A_{5}^{2} \frac{y^{2}}{2}\right]-S \varepsilon^{2}\left[A_{4}^{3} \frac{y^{5}}{20}+A_{4}^{2} A_{5} \frac{y^{4}}{4}+A_{4} A_{5}^{2} \frac{y^{3}}{2}+A_{5}^{3} \frac{y^{2}}{2}\right] \\
& +\varepsilon^{2} A_{4}^{3} \frac{y^{3}}{3}-B r \varepsilon A_{4} \frac{y^{3}}{3}+B r \varepsilon^{2}\left[A_{4}^{2} \frac{y^{4}}{12}+A_{4} A_{5} \frac{y^{3}}{3}\right]+S \varepsilon\left[A_{4}^{2} \frac{y^{4}}{12}+A_{4} A_{5} \frac{y^{3}}{3}\right] \\
& -S \varepsilon^{2}\left[A_{4}^{3} \frac{y^{5}}{30}+A_{4}^{2} A_{5} \frac{y^{4}}{5}+A_{4} A_{5}^{2} \frac{y^{3}}{3}\right]+\varepsilon A_{4} A_{12} y^{2}+B r \frac{d P}{d x} \frac{y^{3}}{3}-B r \lambda A_{4} \frac{y^{3}}{3} \\
& -G r e B r\left[A_{4} \frac{y^{4}}{12}+A_{5} \frac{y^{3}}{3}\right]+B r A_{8} y^{2}-B r \varepsilon \frac{d P}{d x}\left[A_{4} \frac{y^{4}}{6}+A_{5} \frac{y^{3}}{3}\right]+B r \varepsilon^{2} A_{4}^{2} \frac{y^{4}}{24} \\
& +\operatorname{GreBr\varepsilon }\left[A_{4}^{2} \frac{y^{5}}{20}+A_{4} A_{5} \frac{y^{4}}{4}+A_{5}^{2} \frac{y^{3}}{3}\right]+B r \varepsilon \lambda\left[A_{4}^{2} \frac{y^{4}}{6}+A_{4} A_{5} \frac{y^{3}}{3}\right]-B r^{2} \varepsilon \frac{y^{4}}{24} \\
& -B r \varepsilon\left[A_{4} A_{8} \frac{y^{3}}{3}+A_{5} A_{8} y^{2}\right]+B r^{2} \varepsilon^{2}\left[A_{4} \frac{y^{5}}{120}+A_{5} \frac{y^{4}}{24}\right]+B r S \varepsilon\left[A_{4} \frac{y^{5}}{15}+A_{5} \frac{y^{4}}{6}\right] \\
& -B r S \varepsilon^{2}\left[A_{4}^{2} \frac{y^{6}}{360}+A_{4} A_{5} \frac{y^{5}}{60}+A_{5}^{2} \frac{y^{4}}{24}\right]+B r \varepsilon A_{12} \frac{y^{3}}{6}+B r \varepsilon A_{13} \frac{y^{2}}{2}+S \varepsilon A_{4}^{2} \frac{y^{4}}{24} \\
& +S^{2}\left[A_{4} \frac{y^{5}}{120}+A_{5} \frac{y^{4}}{24}\right]-S^{2} \varepsilon\left[A_{4}^{2} \frac{y^{6}}{360}+A_{4} A_{5} \frac{y^{5}}{60}+A_{5}^{2} \frac{y^{4}}{24}\right]+S A_{12} \frac{y^{3}}{6}+S A_{13} \frac{y^{2}}{2} \\
& -S \varepsilon^{2}\left[A_{4}^{3} \frac{y^{5}}{20}+A_{4}^{2} A_{5} \frac{y^{4}}{12}\right]-B r S \varepsilon^{2}\left[A_{4}^{2} \frac{y^{6}}{90}+A_{4} A_{5} \frac{y^{5}}{15}+A_{5}^{2} \frac{y^{4}}{12}\right] \\
& -S^{2} \varepsilon\left[A_{4}^{2} \frac{y^{6}}{90}+A_{4} A_{5} \frac{y^{5}}{15}+A_{5}^{2} \frac{y^{4}}{12}\right]+S^{2} \varepsilon^{2}\left[A_{4}^{3} \frac{y^{7}}{252}+A_{4}^{2} A_{5} \frac{y^{6}}{36}+A_{4} A_{5}^{2} \frac{y^{5}}{12}+A_{5}^{3} \frac{y^{4}}{12}\right] \\
& -S \varepsilon\left[A_{4} A_{12} \frac{y^{4}}{6}+A_{5} A_{12} \frac{y^{3}}{3}\right]-S \varepsilon\left[A_{4} A_{13} \frac{y^{3}}{3}+A_{5} A_{13} y^{2}\right]+A_{20} y+A_{21}, \\
& T_{p_{22}}=A_{22} y+A_{23},
\end{aligned}
$$

where

$$
A_{17}=0,
$$

$$
\begin{aligned}
A_{16}= & \frac{G r e \varepsilon A_{4}^{2}}{24}-\frac{G r e B r}{24}+\operatorname{GreBr\varepsilon }\left[\frac{A_{4}}{120}+\frac{A_{5}}{24}\right]+\operatorname{GreS}\left[\frac{A_{4}}{120}+\frac{A_{5}}{24}\right]+\frac{G r e A_{12}}{6} \\
& +\frac{G r e A_{13}}{2}-\operatorname{GreS\varepsilon }\left[\frac{A_{4}^{2}}{360}+\frac{A_{4} A_{5}}{60}+\frac{A_{5}^{2}}{24}\right]+\frac{\lambda \varepsilon A_{4}^{2}}{6}-\frac{B r \varepsilon}{6}+B r \lambda \varepsilon\left[\frac{A_{4}}{24}+\frac{A_{5}}{6}\right] \\
& +S \lambda\left[\frac{A_{4}}{24}+\frac{A_{5}}{6}\right]-S \varepsilon \lambda\left[\frac{A_{4}^{2}}{60}+\frac{A_{4} A_{5}}{12}+\frac{A_{5}^{2}}{6}\right]+\frac{\lambda A_{12}}{2}-\frac{\lambda A_{4}}{6} \frac{d P}{d x}+\frac{\lambda^{2} A_{4}^{2}}{6} \\
& +G r e \lambda\left[\frac{A_{4}^{2}}{24}+\frac{A_{4} A_{5}}{6}\right]-\frac{\lambda A_{4} A_{8}}{2}-\lambda \frac{d P}{d x}\left[\frac{A_{4}}{6}+\frac{A_{5}}{2}\right]+\operatorname{Gre\lambda }\left[\frac{A_{4}^{2}}{12}+\frac{A_{4} A_{5}}{3}+\frac{A_{5}^{2}}{2}\right] \\
& +\lambda^{2}\left[\frac{A_{4}^{2}}{6}+\frac{A_{4} A_{5}}{2}\right],
\end{aligned}
$$




$$
\begin{aligned}
& A_{20}=\frac{B r \varepsilon}{(1+2 d)}\left[\frac{A_{4}}{6}+\frac{A_{5}}{2}\right]-\frac{\varepsilon^{2}}{(1+2 d)}\left[\frac{A_{4}^{3}}{6}+\frac{A_{4}^{2} A_{5}}{2}\right]-\frac{B r \varepsilon^{2}}{(1+2 d)}\left[\frac{A_{4}^{2}}{12}+\frac{A_{4} A_{5}}{3}+\frac{A_{5}^{2}}{2}\right] \\
& -\frac{S \varepsilon}{(1+2 d)}\left[\frac{A_{4}^{2}}{12}+\frac{A_{4} A_{5}}{3}+\frac{A_{5}^{2}}{2}\right]+\frac{S \varepsilon^{2}}{(1+2 d)}\left[\frac{A_{4}^{3}}{20}+\frac{A_{4}^{2} A_{5}}{4}+\frac{A_{4} A_{5}^{2}}{2}+\frac{A_{5}^{3}}{2}\right] \\
& -\frac{\varepsilon^{2} A_{4}^{3}}{3(1+2 d)}+\frac{B r \varepsilon A_{4}}{3(1+2 d)}-\frac{B r \varepsilon^{2}}{(1+2 d)}\left[\frac{A_{4}^{2}}{12}+\frac{A_{4} A_{5}}{3}\right]-\frac{B r}{(1+2 d)} \frac{d P}{d x} \\
& +\frac{G r e B r}{(1+2 d)}\left[\frac{A_{4}}{12}+\frac{A_{5}}{3}\right]+\frac{B r \lambda A_{4}}{3(1+2 d)}-\frac{B r A_{8}}{(1+2 d)}+\frac{B r \varepsilon}{(1+2 d)} \frac{d P}{d x}\left[\frac{A_{4}}{6}+\frac{A_{5}}{3}\right] \\
& -\frac{G r e B r \varepsilon}{(1+2 d)}\left[\frac{A_{4}^{2}}{20}+\frac{A_{4} A_{5}}{4}+\frac{A_{5}^{2}}{3}\right]-\frac{B r \varepsilon \lambda}{(1+2 d)}\left[\frac{A_{4}^{2}}{6}+\frac{A_{4} A_{5}}{3}\right] \\
& +\frac{B r \varepsilon}{(1+2 d)}\left[\frac{A_{4} A_{8}}{3}+A_{5} A_{8}\right]-\frac{B r \varepsilon^{2} A_{4}^{2}}{24(1+2 d)}+\frac{B r^{2} \varepsilon}{24(1+2 d)}-\frac{B r^{2} \varepsilon^{2}}{(1+2 d)}\left[\frac{A_{4}}{120}+\frac{A_{5}}{24}\right] \\
& -\frac{B r S \varepsilon}{(1+2 d)}\left[\frac{A_{4}}{120}+\frac{A_{5}}{24}\right]+\frac{B r S \varepsilon^{2}}{(1+2 d)}\left[\frac{A_{4}^{2}}{360}+\frac{A_{4} A_{5}}{60}+\frac{A_{5}^{2}}{24}\right]-\frac{B r \varepsilon A_{12}}{6(1+2 d)}-\frac{B r \varepsilon A_{13}}{2(1+2 d)} \\
& -\frac{S \varepsilon A_{4}^{2}}{24(1+2 d)}+\frac{B r S}{24(1+2 d)}-\frac{B r S \varepsilon}{(1+2 d)}\left[\frac{A_{4}}{120}+\frac{A_{5}}{24}\right]-\frac{S^{2}}{(1+2 d)}\left[\frac{A_{4}}{120}+\frac{A_{5}}{24}\right] \\
& +\frac{S^{2} \varepsilon}{(1+2 d)}\left[\frac{A_{4}^{2}}{360}+\frac{A_{4} A_{5}}{60}+\frac{A_{5}^{2}}{24}\right]-\frac{S A_{12}}{6(1+2 d)}-\frac{S A_{13}}{2(1+2 d)}+\frac{S \varepsilon^{2}}{(1+2 d)}\left[\frac{A_{4}^{3}}{20}+\frac{A_{4}^{2} A_{5}}{12}\right] \\
& -\frac{B r S \varepsilon}{(1+2 d)}\left[\frac{A_{4}}{20}+\frac{A_{5}}{12}\right]+\frac{B r S \varepsilon^{2}}{(1+2 d)}\left[\frac{A_{4}^{2}}{90}+\frac{A_{4} A_{5}}{15}+\frac{A_{5}^{2}}{12}\right]+S^{2} \varepsilon\left[\frac{A_{4}^{2}}{90}+\frac{A_{4} A_{5}}{15}+\frac{A_{5}^{2}}{12}\right] \\
& -\frac{S^{2} \varepsilon^{2}}{(1+2 d)}\left[\frac{A_{4}^{3}}{252}+\frac{A_{4}^{2} A_{5}}{36}+\frac{A_{4} A_{5}^{2}}{12}+\frac{A_{5}^{3}}{12}\right]+\frac{S \varepsilon}{(1+2 d)}\left[\frac{A_{4} A_{12}}{6}+\frac{A_{5} A_{12}}{3}\right] \\
& +\frac{S \varepsilon}{(1+2 d)}\left[\frac{A_{4} A_{13}}{3}+A_{5} A_{13}\right]+\frac{2 \varepsilon A_{5} A_{12} d}{(1+2 d)}+\frac{2 \varepsilon A_{4} A_{13} d}{(1+2 d)}-\frac{\varepsilon^{2} d}{(1+2 d)}\left[\frac{A_{4}^{3}}{2}+A_{4}^{2} A_{5}\right] \\
& +\frac{B r \varepsilon d}{(1+2 d)}\left[\frac{A_{4}}{2}+A_{5}\right]-\frac{B r \varepsilon^{2} d}{(1+2 d)}\left[\frac{A_{4}^{2}}{3}+A_{4} A_{5}+A_{5}^{2}\right]-\frac{S \varepsilon d}{(1+2 d)}\left[\frac{A_{4}^{2}}{3}+A_{4} A_{5}+A_{5}^{2}\right] \\
& +\frac{S \varepsilon^{2} d}{(1+2 d)}\left[\frac{A_{4}^{3}}{4}+A_{4}^{2} A_{5}+\frac{3 A_{4} A_{5}^{2}}{2}+A_{5}^{3}\right]+\frac{\varepsilon^{2} A_{4}^{3}}{2(1+2 d)}-\frac{B r \varepsilon A_{4} d}{2(1+2 d)} \\
& +\frac{B r \varepsilon^{2} d}{(1+2 d)}\left[\frac{A_{4}^{2}}{3}+\frac{A_{4} A_{5}}{2}\right]+\frac{S \varepsilon d}{(1+2 d)}\left[\frac{A_{4}^{2}}{3}+\frac{A_{4} A_{5}}{2}\right]-\frac{B r d}{(1+2 d)} \frac{d P}{d x} \\
& -\frac{S \varepsilon^{2} d}{(1+2 d)}\left[\frac{A_{4}^{3}}{4}+\frac{2 A_{4}^{2} A_{5}}{3}+\frac{A_{4} A_{5}^{2}}{2}\right]+\frac{G r e B r d}{(1+2 d)}\left[\frac{A_{4}}{3}+A_{5}\right]+\frac{B r \lambda A_{4} d}{(1+2 d)}-\frac{B r A_{8} d}{(1+2 d)} \\
& +\frac{B r \varepsilon d}{(1+2 d)} \frac{d P}{d x}\left[\frac{2 A_{4}}{3}+A_{5}\right]-\frac{G r e B r \varepsilon d}{(1+2 d)}\left[\frac{A_{4}^{2}}{4}+A_{4} A_{5}+A_{5}^{2}\right]-\frac{B r \varepsilon \lambda d}{(1+2 d)}\left[\frac{2 A_{4}^{2}}{3}+A_{4} A_{5}\right] \\
& +\frac{B r \varepsilon d}{(1+2 d)}\left[A_{4} A_{8}+2 A_{5} A_{8}\right]-\frac{B r \varepsilon^{2} A_{4}^{2} d}{6(1+2 d)}+\frac{B r^{2} \varepsilon d}{6(1+2 d)}-\frac{B r^{2} \varepsilon^{2} d}{(1+2 d)}\left[\frac{A_{4}}{24}+\frac{A_{5}}{6}\right] \\
& -\frac{B r S \varepsilon d}{(1+2 d)}\left[\frac{A_{4}}{24}+\frac{A_{5}}{6}\right]+\frac{B r S \varepsilon^{2} d}{(1+2 d)}\left[\frac{A_{4}^{2}}{60}+\frac{A_{4} A_{5}}{12}+\frac{A_{5}^{2}}{6}\right]-\frac{B r \varepsilon A_{12} d}{2(1+2 d)}-\frac{B r \varepsilon A_{13} d}{(1+2 d)} \\
& -\frac{S \varepsilon A_{4}^{2} d}{6(1+2 d)}+\frac{B r S d}{6(1+2 d)}-\frac{B r S \varepsilon d}{(1+2 d)}\left[\frac{A_{4}}{24}+\frac{A_{5}}{6}\right]-\frac{S^{2} d}{(1+2 d)}\left[\frac{A_{4}}{24}+\frac{A_{5}}{6}\right] \\
& +\frac{S^{2} \varepsilon d}{(1+2 d)}\left[\frac{A_{4}^{2}}{60}+\frac{A_{4} A_{5}}{12}+\frac{A_{5}^{2}}{6}\right]-\frac{S A_{12} d}{2(1+2 d)}-\frac{S A_{13} d}{(1+2 d)}+\frac{S \varepsilon^{2} d}{(1+2 d)}\left[\frac{A_{4}^{3}}{4}+\frac{A_{4}^{2} A_{5}}{3}\right]
\end{aligned}
$$




$$
\begin{aligned}
& -\frac{B r S \varepsilon d}{(1+2 d)}\left[\frac{A_{4}}{4}+\frac{A_{5}}{3}\right]+\frac{B r S \varepsilon^{2} d}{(1+2 d)}\left[\frac{A_{4}^{2}}{15}+\frac{A_{4} A_{5}}{3}+\frac{A_{5}^{2}}{3}\right]+\frac{S^{2} \varepsilon d}{(1+2 d)}\left[\frac{A_{4}^{2}}{15}+\frac{A_{4} A_{5}}{3}+\frac{A_{5}^{2}}{3}\right] \\
& -\frac{S^{2} \varepsilon^{2} d}{(1+2 d)}\left[\frac{A_{4}^{3}}{36}+\frac{A_{4}^{2} A_{5}}{6}+\frac{5 A_{4} A_{5}^{2}}{12}+\frac{A_{5}^{3}}{3}\right]+\frac{S \varepsilon d}{(1+2 d)}\left[\frac{2 A_{4} A_{12}}{3}+A_{5} A_{12}\right] \\
& +\frac{S \varepsilon d}{(1+2 d)}\left[A_{4} A_{13}+2 A_{5} A_{13}\right]+\frac{\varepsilon^{2} A_{4}^{2} A_{5} d}{(1+2 d)}-\frac{B r \varepsilon A_{4} A_{5} d}{(1+2 d)}+\frac{B r \varepsilon^{2} d}{(1+2 d)}\left[\frac{A_{4} A_{5}}{2}+A_{5}^{2}\right] \\
& +\frac{S \varepsilon d}{(1+2 d)}\left[\frac{A_{4} A_{5}}{2}+A_{5}^{2}\right]-\frac{S \varepsilon^{2} d}{(1+2 d)}\left[\frac{A_{4}^{2} A_{5}}{3}+A_{4} A_{5}^{2}+A_{5}^{3}\right]+\frac{\varepsilon A_{5} A_{12} d}{(1+2 d)}+\frac{\varepsilon A_{4} A_{13} d}{(1+2 d)}, \\
& A_{18}=A_{20}-\varepsilon A_{5} A_{12}-\varepsilon A_{4} A_{13}, \\
& A_{19}=A_{18}, \\
& A_{21}=A_{19}, \\
& A_{22}=\varepsilon^{2}\left[\frac{A_{4}^{3}}{2}+A_{4}^{2} A_{5}\right]-\operatorname{Br\varepsilon }\left[\frac{A_{4}}{2}+A_{5}\right]+\operatorname{Br}^{2}\left[\frac{A_{4}^{2}}{3}+A_{4} A_{5}+A_{5}^{2}\right]+\varepsilon^{2} A_{4}^{3} \\
& +S \varepsilon\left[\frac{A_{4}^{2}}{3}+A_{4} A_{5}+A_{5}^{2}\right]-S \varepsilon^{2}\left[\frac{A_{4}^{3}}{4}+A_{4}^{2} A_{5}+\frac{3 A_{4} A_{5}^{2}}{2}+A_{5}^{3}\right]-B r \varepsilon A_{4} \\
& +B r \varepsilon^{2}\left[\frac{A_{4}^{2}}{3}+A_{4} A_{5}\right]+S \varepsilon\left[\frac{A_{4}^{2}}{3}+A_{4} A_{5}\right]-S \varepsilon^{2}\left[\frac{A_{4}^{3}}{6}+\frac{2 A_{4}^{2} A_{5}}{3}+A_{4} A_{5}^{2}\right] \\
& +B r \frac{d P}{d x}-\operatorname{GreBr}\left[\frac{A_{4}}{3}+A_{5}\right]-B r \lambda A_{4}+3 B r A_{8}-B r \varepsilon \frac{d P}{d x}\left[\frac{2 A_{4}}{3}+A_{5}\right] \\
& +\operatorname{GreBr\varepsilon }\left[\frac{A_{4}^{2}}{4}+A_{4} A_{5}+A_{5}^{2}\right]+\operatorname{Br\varepsilon } \lambda\left[\frac{2 A_{4}^{2}}{3}+A_{4} A_{5}\right]-\operatorname{Br\varepsilon }\left[A_{4} A_{8}+2 A_{5} A_{8}\right] \\
& +\frac{B r \varepsilon^{2} A_{4}^{2}}{6}-\frac{B r^{2} \varepsilon}{6}+B r^{2} \varepsilon^{2}\left[\frac{A_{4}}{24}+\frac{A_{5}}{6}\right]+B r S \varepsilon\left[\frac{A_{4}}{24}+\frac{A_{5}}{6}\right]+\frac{B r \varepsilon A_{12}}{2} \\
& -B r S \varepsilon^{2}\left[\frac{A_{4}^{2}}{60}+\frac{A_{4} A_{5}}{12}+\frac{A_{5}^{2}}{6}\right]+B r \varepsilon A_{13}+\frac{S \varepsilon A_{4}^{2}}{6}-\frac{B r S}{6}+B r S \varepsilon\left[\frac{A_{4}}{24}+\frac{A_{5}}{6}\right] \\
& +S^{2}\left[\frac{A_{4}}{24}+\frac{A_{5}}{6}\right]-S^{2} \varepsilon\left[\frac{A_{4}^{2}}{60}+\frac{A_{4} A_{5}}{12}+\frac{A_{5}^{2}}{6}\right]+\frac{S A_{12}}{2}+S A_{13}-S \varepsilon^{2}\left[\frac{A_{4}^{3}}{4}+\frac{A_{4}^{2} A_{5}}{3}\right] \\
& +B r S \varepsilon\left[\frac{A_{4}}{4}+\frac{A_{5}}{3}\right]-B r S \varepsilon^{2}\left[\frac{A_{4}^{2}}{15}+\frac{A_{4} A_{5}}{3}+\frac{A_{5}^{2}}{3}\right]-S^{2} \varepsilon\left[\frac{A_{4}^{2}}{15}+\frac{A_{4} A_{5}}{3}+\frac{A_{5}^{2}}{3}\right] \\
& +S^{2} \varepsilon^{2}\left[\frac{A_{4}^{3}}{36}+\frac{A_{4}^{2} A_{5}}{6}+\frac{5 A_{4} A_{5}^{2}}{12}+\frac{A_{5}^{3}}{3}\right]-S \varepsilon\left[\frac{2 A_{4} A_{12}}{3}+A_{5} A_{12}\right] \\
& -S \varepsilon\left[A_{4} A_{13}+2 A_{5} A_{13}\right]-\frac{3 \varepsilon^{2} A_{4}^{3}}{2}+\frac{3 B r \varepsilon A_{4}}{2}-B r \varepsilon^{2}\left[\frac{2 A_{4}^{2}}{3}+\frac{3 A_{4} A_{5}}{2}\right] \\
& -S \varepsilon\left[\frac{2 A_{4}^{2}}{3}+\frac{3 A_{4} A_{5}}{2}\right]+S \varepsilon^{2}\left[\frac{5 A_{4}^{3}}{12}+\frac{4 A_{4}^{2} A_{5}}{3}+\frac{3 A_{4} A_{5}^{2}}{2}\right]-\varepsilon^{2} A_{4}^{2} A_{5}+B r \varepsilon A_{4} A_{5} \\
& -B r \varepsilon^{2}\left[\frac{A_{4} A_{5}}{2}+A_{5}^{2}\right]-S \varepsilon\left[\frac{A_{4} A_{5}}{2}+A_{5}^{2}\right]+S \varepsilon^{2}\left[\frac{A_{4}^{2} A_{5}}{3}+A_{4} A_{5}^{2}+A_{5}^{3}\right] \\
& \text { - } \varepsilon A_{5} A_{12}-\varepsilon A_{4} A_{13} \text {, }
\end{aligned}
$$

$$
A_{23}=-(1+d) A_{22} \text {. }
$$

Now, the approximate solution of the momentum and energy eqns(7) and (9) of the fluid as $p \rightarrow 1$ are:

$$
u=u_{0}+u_{1}+u_{2}+\ldots
$$




$$
T_{f}=T_{f_{0}}+T_{f_{1}}+T_{f_{2}}+\ldots
$$

The interesting phenomena to be investigated in this study are onset of reverse flow, pressure gradient, skin friction, rate of heat transfer between the fluid and boundary plates and the pressure gradient.

In order to obtain the pressure gradient required to drive the flow formation for a constant mass flux $q$, the integral

$$
\int_{0}^{1} u d y=q
$$

is evaluated for different flow parameters (see table 1).

The pressure gradient can be expressed as:

$$
\frac{d P}{d x}=\frac{X_{1}}{X_{2}}
$$

where

$$
\begin{aligned}
X_{1}= & \frac{1}{2}+\text { Gre }\left[\frac{A_{4}}{24}+\frac{A_{5}}{12}\right]+\frac{\lambda A_{4}}{12}+\frac{G r e \varepsilon A_{4}^{2}}{80}-\frac{G r e B r}{80}+\text { GreBre }\left[\frac{A_{4}}{360}+\frac{A_{5}}{80}\right] \\
& +\operatorname{GreS}\left[\frac{A_{4}}{360}+\frac{A_{5}}{80}\right]-\operatorname{GreS\varepsilon }\left[\frac{A_{4}^{2}}{1008}+\frac{A_{4} A_{5}}{180}+\frac{A_{5}^{2}}{80}\right]+\frac{A_{12} G r e}{24}+\frac{A_{13} G r e}{12}+\frac{\lambda \varepsilon A_{4}^{2}}{24} \\
& -\frac{B r \lambda}{24}+\operatorname{Br} \lambda \varepsilon\left[\frac{A_{4}}{80}+\frac{A_{5}}{24}\right]+S \lambda\left[\frac{A_{4}}{80}+\frac{A_{5}}{24}\right]-S \varepsilon \lambda\left[\frac{A_{4}^{2}}{180}+\frac{A_{4} A_{5}}{40}+\frac{A_{5}^{2}}{24}\right] \\
& +\frac{A_{12} \lambda}{12}-\frac{G r e \lambda A_{4}^{2}}{720}+\operatorname{Gre\lambda }\left[\frac{A_{4}^{2}}{40}+\frac{A_{4} A_{5}}{12}+\frac{A_{5}^{2}}{12}\right]+\lambda^{2}\left[\frac{A_{4}^{2}}{24}+\frac{A_{4} A_{5}}{12}\right]-q,
\end{aligned}
$$

and

$$
X_{2}=\frac{1}{12}+\frac{\lambda A_{4}}{24}+\frac{\lambda A_{5}}{12},
$$

The critical values of mixed convection parameter Gre which signalled the onset of reverse flow at $y=1$, can be obtained from the turning point of $u$, i.e $\left.\frac{d u}{d y}\right|_{y=1}=0$. Therefore, the influence of different parameters as they control the critical Gre are presented in table 2.

The skin friction at the plate surfaces can be defined as:

$$
\tau_{(0,1)}=\left.\mu^{\star} \frac{d u^{\star}}{d y^{\star}}\right|_{y^{\star}=0, h}
$$

where $\mu^{\star}$ is the dimensional variable viscosity. Thus, the local skin friction factor can be expressed as:

$$
\tau=\frac{\tau_{(0,1)} h}{\mu_{0} U}=\left.\left(1-\lambda T_{f}\right) \frac{d u}{d y}\right|_{y=0,1}
$$

while the local surface heat flux is expressed as:

$$
(0,1)=-\left.k^{\star} \frac{d T_{f}^{\star}}{d y^{\star}}\right|_{y^{\star}=0, h}
$$

where $k^{\star}$ is the dimensional variable thermal conductivity, together with the expression of Nusselt number,

$$
N u_{h}=\frac{l_{(0,1)}}{\left(T_{w}-T_{0}\right)} \frac{h}{k_{0}} .
$$

Therefore, the Nusselt number can be expressed as:

$$
N u=-\left.\left(1-\varepsilon T_{f}\right) \frac{d T_{f}}{d y}\right|_{y=0,1}
$$




\section{Validation}

Setting $B r=0, \lambda=0, \varepsilon=0, \frac{d P}{d x}=0$ and $d=0$, the work of Jha and Ajibade [55] is recovered, see table 5 .

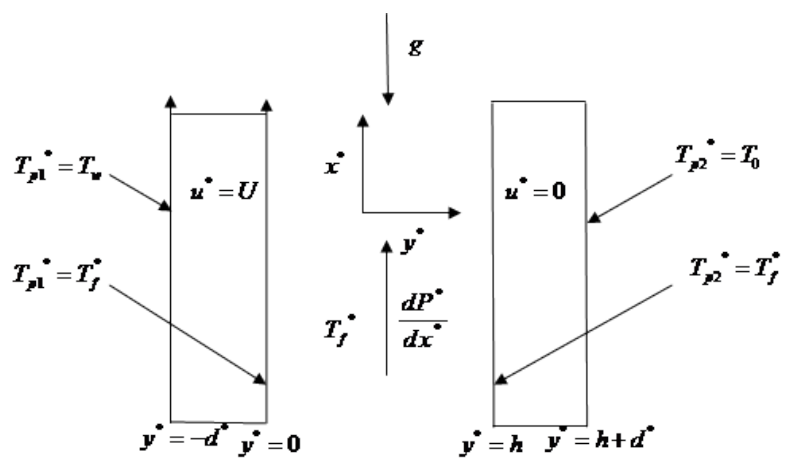

Figure 1: Schematic diagram of the problem

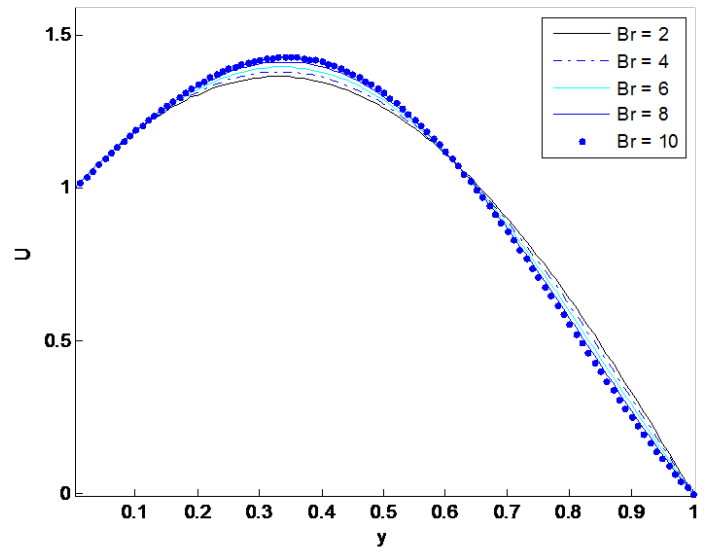

Figure 2: Velocity profile for different values of Brinkman number $\mathrm{Br}$ $(S=0.4$, Gre $=10, \lambda=-0.3, \varepsilon=-0.3, d=0.2, q=1)$

\section{Results and discussion}

Viscous dissipation and boundary thickness effects on a steady mixed convection flow in the presence of variable viscosity and thermal conductivity have been theoretically analysed. The flow is governed by six basic parameters, which are: Gre, thermal buoyancy, $S$ heat generating/absorbing, $\lambda$, viscosity variation parameter

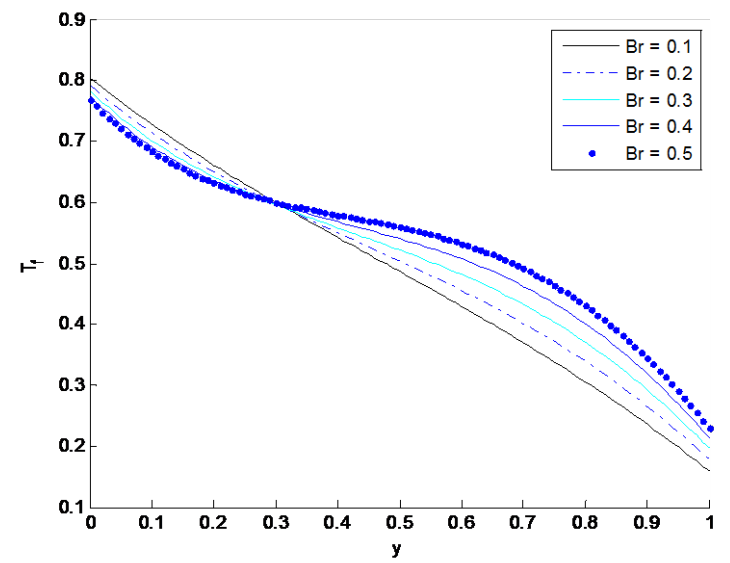

Figure 3: Temperature profile for different values of Brinkman number $\operatorname{Br}(S=0.4$, Gre $=10, \lambda=-0.3, \varepsilon=-0.3, d=0.2, q=1)$

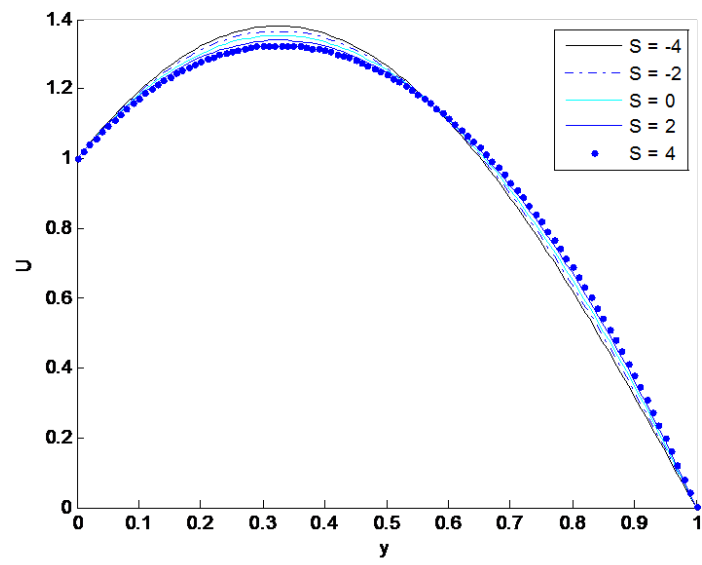

Figure 4: Velocity profile for different values of heat source/sink parameter $S(B r=0.2$, Gre $=10, \lambda=-0.3, \varepsilon=-0.3, d=0.2, q=1)$

and $\varepsilon$, thermal conductivity variation parameter, $\mathrm{Br}$ Brinkman number, $q$ mass flux and $d$ the boundary thickness. The value of $P r$ is chosen to be 0.71 throughout the work for air as the working fluid. The solutions for momentum and energy equations in the fluid section have been simulated for carefully selected values of the controlling parameters and the results are presented in figures 2-15. Also, the pressure gradient required to drive the flow to a prescribed mass flux is presented in table 1 while the critical values of the mixed convection parameter after which flow reversal is achieved near the stationary plate is given in table 2 . The skin friction and the rate of heat transfer on the fluid-plate interface were tabulated in tables 3 and 4.

Figures 2 and 3 show the effects of Brinkman number $\mathrm{Br}$ 
Table 1: Variation of pressure gradient $\left(\frac{d P}{d x}\right)$ for different values of $S, q, d$ and $G r e$ where $\lambda=-0.3, \varepsilon=-0.3, B r=0.2$

\begin{tabular}{cc|ccc}
\hline$S$ & $q$ & $(d=0.2$, Gre $=10)$ & $(d=0.2$, Gre $=20)$ & $(d=0.5$, Gre $=20)$ \\
& & $\frac{d P}{d x}$ & $\frac{d P}{d x}$ & $\frac{d P}{d x}$ \\
\hline-0.4 & -0.6376 & 5.5742 & 7.0079 \\
-0.2 & & -0.9155 & 5.0210 & 6.0491 \\
0 & 1 & -1.1935 & 4.4677 & 5.0903 \\
0.2 & & -1.4715 & 3.9145 & 4.1316 \\
0.4 & -1.7494 & 3.3612 & 3.1728 \\
\hline-0.4 & -14.7532 & -8.5434 & -7.1098 \\
-0.2 & & -15.0332 & -9.0967 & -8.0686 \\
0 & 2 & -15.3112 & -9.6499 & -9.0273 \\
0.2 & -15.5891 & -10.2032 & -9.9861 \\
0.4 & -15.8671 & -10.7565 & -10.9449 \\
\hline
\end{tabular}

Table 2: Critical values of Gre at the stationary plate $y=1$ for different values of $S, \lambda, d$ and $\varepsilon$ where $q=1$ and $B r=0.2$

\begin{tabular}{cc|ccc}
\hline$S$ & $\lambda$ & $(d=0.2, \varepsilon=-0.2)$ & $(d=0.2, \varepsilon=-0.4)$ & $(d=0.5, \varepsilon=-0.4)$ \\
Gre $_{1}$ & Gre $_{1}$ & \\
\hline-0.4 & & 54.57910812 & 52.84378325 & 67.22029661 \\
-0.2 & & 56.09504727 & 54.43472074 & 70.01808552 \\
0 & 0.2 & 57.69881288 & 56.12581355 & 73.06158621 \\
0.2 & & 59.39826494 & 57.92682695 & 76.38465434 \\
0.4 & & 61.20223028 & 59.84883818 & 80.02766446 \\
\hline-0.4 & & 61.51931314 & 59.59863180 & 75.71150476 \\
-0.2 & & 64.02558277 & 62.22361979 & 80.59857570 \\
0 & 0.4 & 66.74858497 & 65.09490123 & 86.16966963 \\
0.2 & & 69.71770381 & 68.24884547 & 92.57920319 \\
0.4 & & 72.96788618 & 71.72935347 & 100.0318533 \\
\hline
\end{tabular}

Table 3: skin friction $\tau$ at the heated plate $y=0$ and cold plate $y=1$, for different values of $S, d$ and $\lambda$ where $B r=0.2, \varepsilon=-0.3, G r e=10$ and $q=1$

\begin{tabular}{|c|c|c|c|c|c|c|}
\hline \multirow{2}{*}{$S$} & \multicolumn{2}{|c|}{$(d=0.2, \lambda=-0.2)$} & \multicolumn{2}{|c|}{$(d=0.2, \lambda=-0.4)$} & \multicolumn{2}{|c|}{$(d=0.5, \lambda=-0.4)$} \\
\hline & $\tau_{\mathrm{C}}$ & $\tau_{1}$ & $\tau_{0}$ & $\tau_{1}$ & $\tau_{0}$ & $\tau_{1}$ \\
\hline-0.4 & 0.5218 & 3.5129 & 2.2957 & 3.6098 & 2.5932 & 4.3377 \\
\hline-0.2 & 0.3835 & 3.3964 & 2.1362 & 3.4779 & 2.3013 & 4.0554 \\
\hline 0 & 2.2464 & 3.2811 & 1.9789 & 3.3484 & 2.0196 & 3.7858 \\
\hline 0.2 & 2.1104 & 3.1670 & 1.8236 & 3.2212 & 1.7470 & 3.5279 \\
\hline 0.4 & 1.9755 & 3.0539 & 1.6703 & 3.0961 & 1.4820 & 3.2806 \\
\hline
\end{tabular}


Table 4: Rate of heat transfer at the heated plate $y=0$ and cold plate $y=1$ for different values of $S, d$ and $\varepsilon$ where $B r=0.2, G r e=10, q=1$ and $\lambda=-0.2$

\begin{tabular}{c|cccccc}
\hline & $(d=0.2, \varepsilon=-0.2)$ & $(d=0.2, \varepsilon=-0.4)$ & $(d=0.5, \varepsilon=-0.4)$ \\
$S$ & \multicolumn{7}{|c}{$N u_{1}$} & $N u_{0}$ & $N u_{1}$ & $N u_{0}$ & $N u_{1}$ \\
& $N u_{0}$ & $N u_{1}$ & 0.7986 & 1.1820 & 0.4326 & 0.9635 \\
-0.4 & 0.6852 & 1.1133 & 0.861 & 1.1139 & 0.5320 & 0.8638 \\
-0.2 & 0.7643 & 1.0529 & 0.8861 & 1.0532 & 0.6143 & 0.7807 \\
0 & 0.8370 & 0.9976 & 0.9639 & 1.053 \\
0.2 & 0.9034 & 0.9476 & 1.0321 & 0.9997 & 0.6805 & 0.7133 \\
0.4 & 0.9635 & 0.9027 & 1.0911 & 0.9533 & 0.7316 & 0.6608 \\
\hline
\end{tabular}

Table 5: Comparison of present work and Jha and Ajibade (2010)

\begin{tabular}{|c|c|c|c|c|}
\hline \multirow[t]{2}{*}{$S$} & \multicolumn{2}{|c|}{$\begin{array}{l}\text { Jha and Ajibade (2010) } \\
\qquad G r=10, y=0.5\end{array}$} & \multicolumn{2}{|c|}{$\begin{array}{c}\text { Present Work } \\
\text { Gre }=10, y=0.5, B r=\frac{d P}{d x}=d=\lambda=\varepsilon=0\end{array}$} \\
\hline & Velocity & Temperature & Velocity & Temperature \\
\hline 1 & 1.0659 & 0.4434 & 1.0599 & 0.4440 \\
\hline 0.5 & 1.0940 & 0.4703 & 1.0924 & 0.4704 \\
\hline-0.5 & 1.1593 & 0.5330 & 1.1576 & 0.5329 \\
\hline-1 & 1.1975 & 0.5697 & 1.1901 & 0.5690 \\
\hline
\end{tabular}

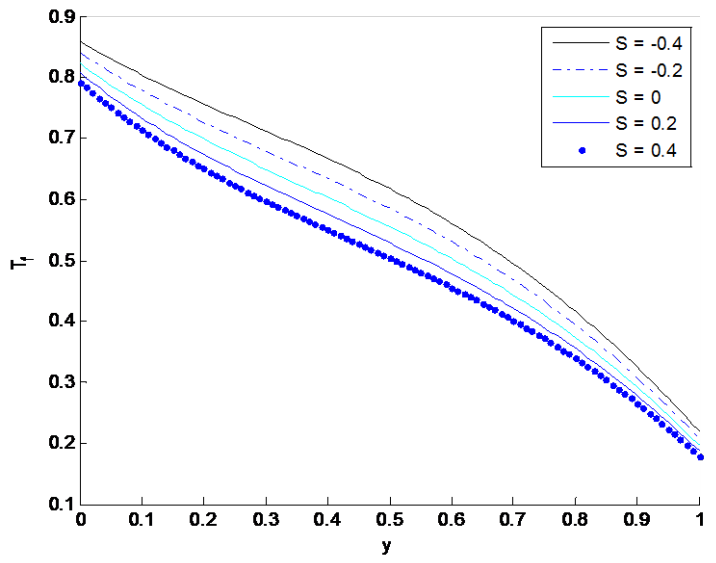

Figure 5: Temperature profile for different values of heat source/sink parameter $S(B r=0.2$, Gre $=10, \lambda=-0.3, \varepsilon=-0.3$, $d=0.2, q=1)$

on velocity and temperature distributions. It is seen that the velocity increases at the heated plate with increase in $B r$. This is due to the fact that, Brinkman number is the ratio of heat produced by viscous dissipation to heat transported by molecular conduction. However, a decrease is observed near the cold plate with growing $\mathrm{Br}$. This clearly reveals the effect of the pressure gradient and the impulsive motion by the heated plate to increase the velocity near the plate $y=0$. On the other hand, figure 3 reveals that the temperature distribution decreases with increase in Brinkman number near the heated plate while

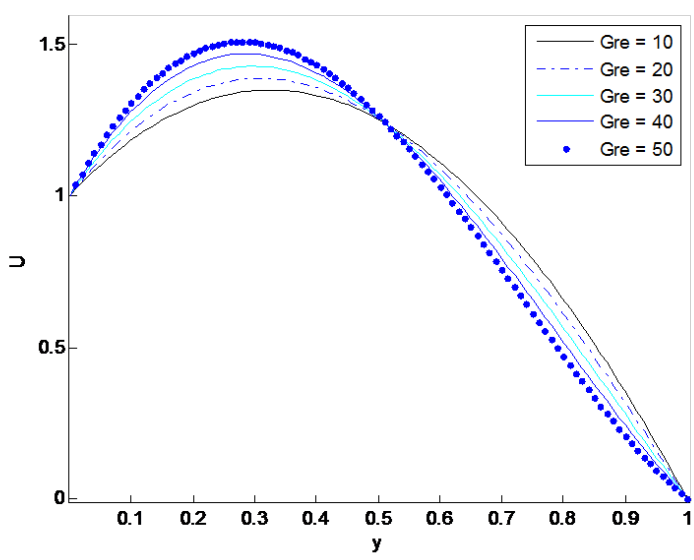

Figure 6: Velocity profile for different values of mixed convection parameter $\mathrm{Gre}(\mathrm{Br}=0.2, S=0.4, \lambda=-0.3, \varepsilon=-0.3, d=0.2$, $q=1)$

an increase is observed near the cold plate as a result of energy diffusion towards the cold plate which increases the temperature of the fluid. It is also observed that the inter-facial temperature decreases at the heated plate with an increase in $\mathrm{Br}$ while an increase is observed near the cold plate. The effect of $\mathrm{Br}$ is more pronounced at the cold, thus increases the temperature at the region.

Figures 4 and 5 show the effects of heat generating/absorption parameter $S$ on velocity and temperature distributions. The velocity increases with increase in heat generation $S<0$ near the heated plate while it decreases 


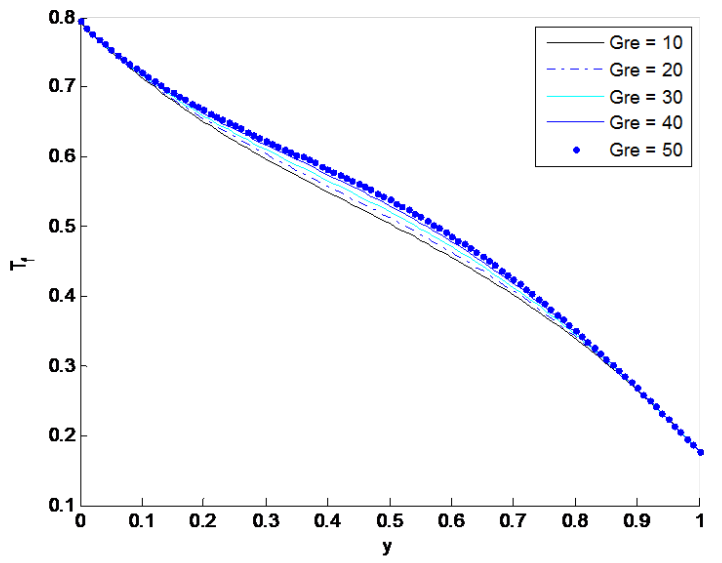

Figure 7: Temperature profile for different values of mixed convection parameter $\mathrm{Gre}(\mathrm{Br}=0.2, S=0.4, \lambda=-0.3, \varepsilon=-0.3, d=0.2$, $q=1)$

near the cold plate. However, near the heated plate, the velocity distribution decreases with increase in heat absorption $S>0$ due to the fact that the heat generated is absorbed by the fluid particles which makes the fluid more dense, thus reduces the temperature and slow down the fluid flow. It is also observed that the temperature distribution increases across the channel with increase in heat generation $S<0$. This is physically true since heat generated strengthens the convection current of the fluid thereby reduces the density of the fluid, thus increases the fluid temperature. This is attributed to the fact that, viscous dissipation, wall conduction and thermal conductivity add more heat to the fluid and the heat diffuses by pressure gradient. It is further noticed that the temperature distribution decreases with increase in heat absorption $S>0$. This physically true, when heat is absorbed, then the fluid becomes dense and the temperature reduces. The effect of $S$ on the inter-facial temperature is more pronounced at the heated plate than the cold plate.

Figures 6 and 7 show the effects of mixed convection Gre on the velocity and temperature distributions respectively. The velocity profile increases with increase in Gre near the heated plate. This is attributed to the role of heat generated from the wall and the internal heat generated which makes the fluid less dense and moves easily by the buoyancy forces. Near the cold plate, it has been observed that the velocity distribution decreases with increase in Gre as the fluid is more dense near the cold plate. On figure 7, the temperature profile increases with increase in Gre. This is physically true, since the heat generated by wall conduction and viscous dissipation diffuses by the

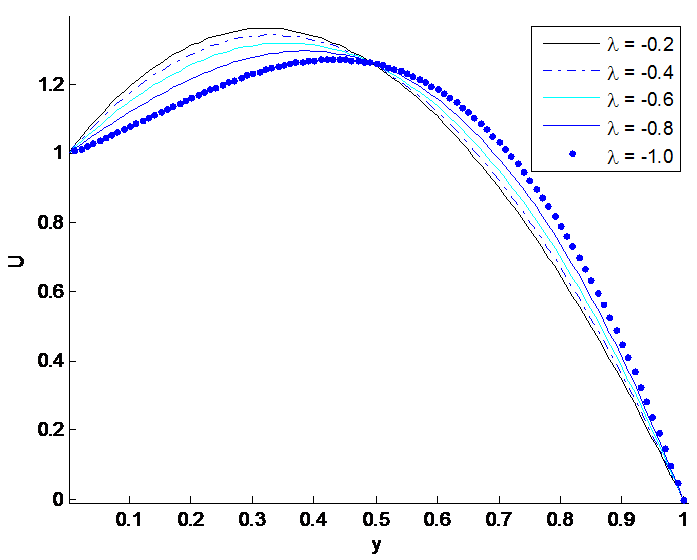

Figure 8: Velocity profile for different values of variable viscosity $\lambda$ $(B r=0.2, S=0.4$, Gre $=10, \varepsilon=-0.3, d=0.2, q=1)$

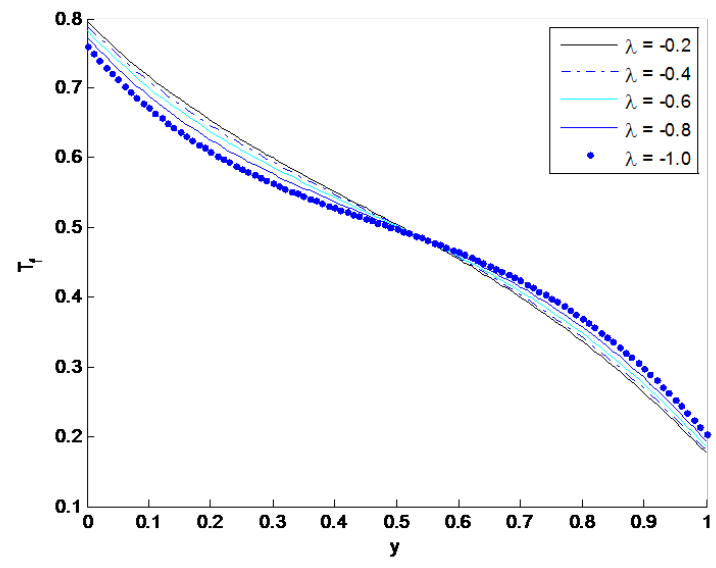

Figure 9: Temperature profile for different values of variable viscosity $\lambda(B r=0.2, S=0.4$, Gre $=10, \varepsilon=-0.3, d=0.2, q=1)$

buoyancy forces which strengthens convection current, which in return increases fluid velocity and consequently the sheared heating at every fluid sections. The effect of Gre on the inter-facial temperature is insignificant at the plates.

The effects of variable viscosity $\lambda$ on velocity and temperature distributions are captured on figures 8 and 9 . It is observed from figure 8 that the velocity distribution decreases near the heated plate with increase in viscosity while an increase has been observed near the cold plate. Decrease in velocity near the heated plate is due to the fact that there is an increase in convection current which in return increases the viscosity of the fluid, thereby increasing the viscous drag that decreases the velocity. However, the increase of velocity near the cold plate is 


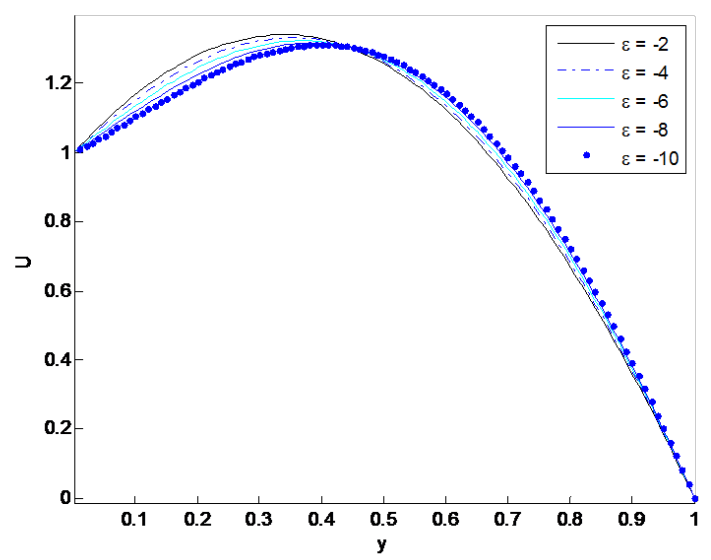

Figure 10: Velocity profile for different values of variable thermal conductivity $\varepsilon(B r=0.2, S=0.4$, Gre $=10, \lambda=-0.3, d=0.2$, $q=1)$

attributed to the decrease in heat which reduces the fluid viscosity, and eventually increases the fluid velocity. The temperature distribution decreases near the heated plate with increase in viscosity $\lambda$ while it increases near the cold plate. The change in viscosity affects the inter-facial temperature on both the plates. It can be seen that the temperature at the heated plate decreases with increase in viscosity while a reverse case was observed at the cold plate.

The effects of thermal conductivity $\varepsilon$ on velocity and temperature are depicted on figures 10 and 11. It is observed that the velocity distribution decreases with increase in thermal conductivity near the heated plate while it increases near the cold plate. On the other hand, the temperature distribution decreases with increase in thermal conductivity. This is attributed to increase in thermal conductivity which enhances heat transport within the system so that heat from the internal heating mechanism diffuses towards the cold plate at a rate proportional to the thermal conductivity. Consequently, convection current is weakened near the heated plate and strengthened near the cold plate. The effect of the thermal conductivity on the inter-facial temperature is little bit pronounced at the cold plate than the heated plate as a result of the energy generated which diffuses towards the plate thus increases the temperature.

Figures 12 and 13 show the different boundary layer thickness $d$ effects on velocity and temperature distributions. It can be seen from figure 12 that the velocity distribution decreases with increase in boundary plate thickness $d$ near the heated plate. This is physically true, since when the thickness of a material is increased, the heat flux

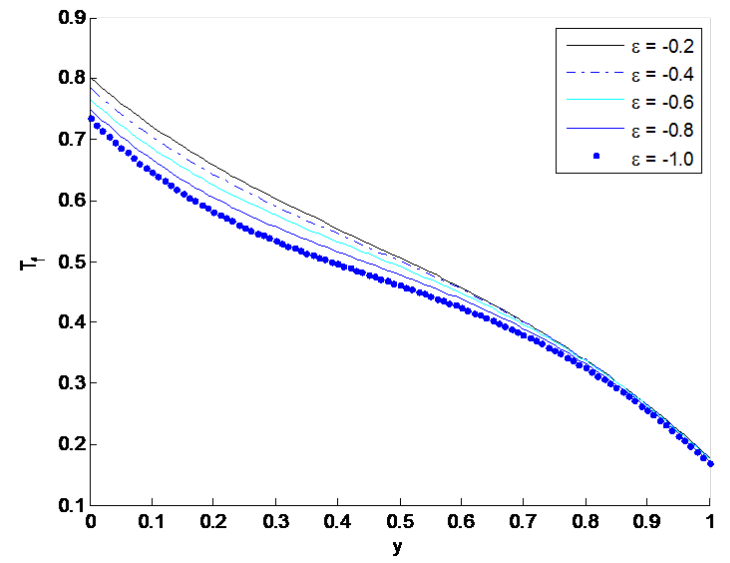

Figure 11: Temperature profile for different values of variable thermal conductivity $\varepsilon(B r=0.2, S=0.4$, Gre $=10, \lambda=-0.3, d=0.2$, $q=1)$

reduces and cools down the fluid. Towards the cold plate, the velocity distribution increases. This is attributed to the fact that the energy dissipated diffuses towards the cold plate which strengthens the convection current, hence increases the fluid flow. The temperature distribution decreases near the heated plate with increase in boundary plate thickness. This is due to the fact that, increase in the thickness of a material reduces the heat flux through the system, which causes decrease in the temperature of the fluid. It is also observed that the boundary plate thickness has more effect on the inter-facial temperature at the heated plate than the cold plate.

Figures 14 and 15 display the velocity and temperature distributions for different values of mass flux $q$. The velocity distribution increases with increasing $q$. This is due to the fact that, increase in mass flux is attributed to increase in pressure gradient to boost the fluid particles' flow. The temperature distribution decreases near the heated plate with increase in $q$ while a reverse case is observed near the cold plate. Since the mass flux increases with growing pressure gradient, the effect of natural convection decreases so as to maintain the required flux within the channel. However, the effect of channel boundary motion is not there so that an increase is required in fluid temperature to drive the flow to the prescribed flow.

Table 1 shows the effect of dimensionless flow parameters on pressure gradient in the channel. It is observed that the pressure gradient increases with increase in mass flux $q$. That is, an increase in mass flux requires a corresponding increase in pressure gradient to drive the flow. This is obtained when Gre is relatively small. However, when Gre is large, the natural convection dominated 


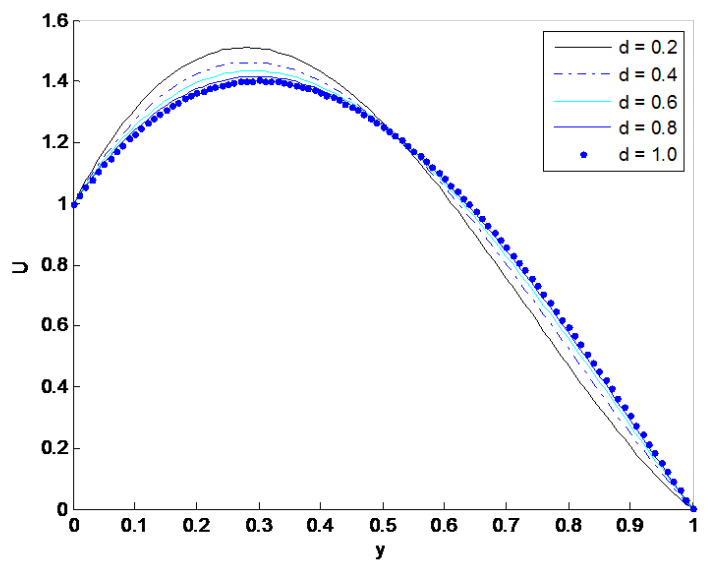

Figure 12: Velocity profile for different values of boundary thickness $d(B r=0.2, S=0.4$, Gre $=50, \lambda=-0.3, \varepsilon=-0.3, q=1)$

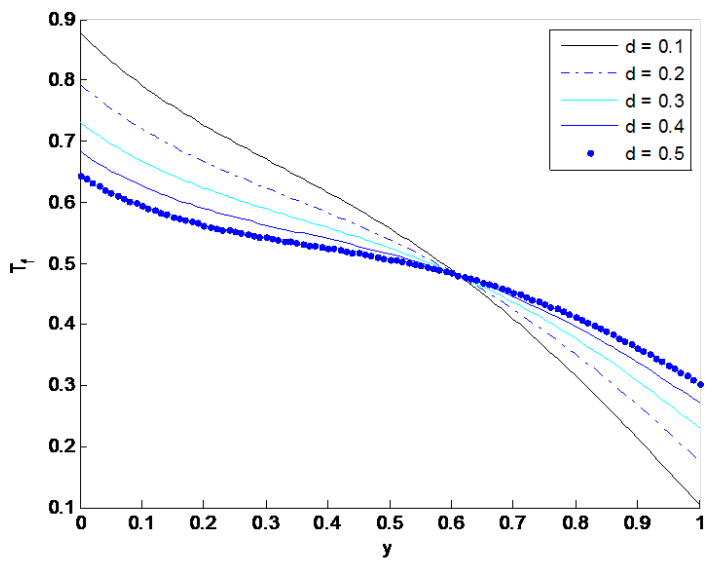

Figure 13: Temperature profile for different values of boundary thickness $d(B r=0.2, S=0.4, G r e=50, \lambda=-0.3, \varepsilon=-0.3$, $q=1)$

the heat transfer, pressure gradient acts against the flow so as to obtain the required mass flux, thus the positive values of pressure gradient at large Gre. The table also shows that, a decrease in pressure gradient is obtained by increasing heat absorption $S<0$. This is due to the fact that, increase in heat generation leads to increase in convection current by buoyancy forces. It is further observed that the pressure gradient acts against the flow when the boundary thickness is increased for relatively small mass flux. However, when the mass is high, the required pressure gradient decreases for a corresponding increase in boundary thickness.

Table 2 shows the critical values of Gre which signal the onset of reverse flow at the cold plate $y=1$. It can be seen that smaller critical values can be obtained by en-

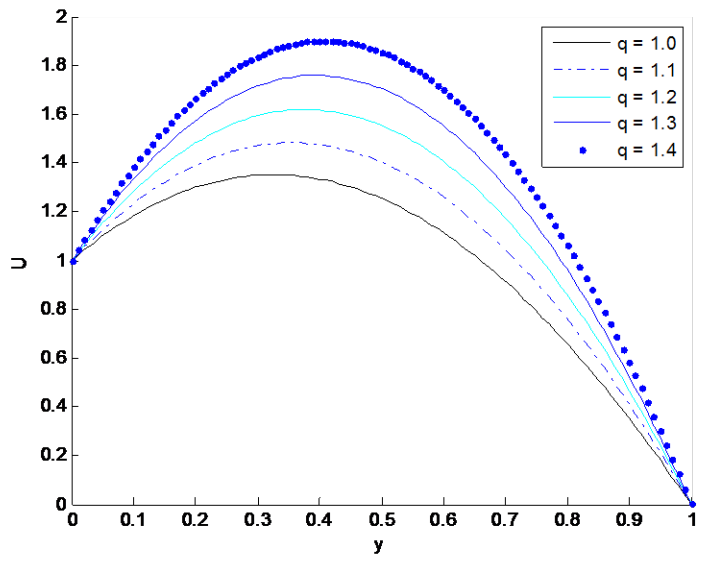

Figure 14: Velocity profile for different values of mass flux $q$ ( $\mathrm{Br}=$ $0.2, S=0.4$, Gre $=10, \lambda=-0.3, \varepsilon=-0.3, d=0.2$ )

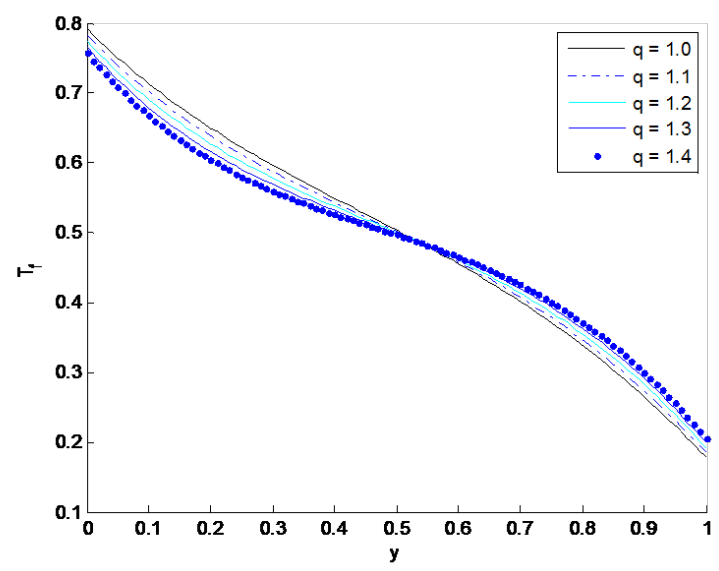

Figure 15: Temperature profile for different values of mass flux $q$ $(B r=0.2, S=0.4, G r e=10, \lambda=-0.3, \varepsilon=-0.3, d=0.2)$

hancing the thermal conductivity $\varepsilon$ or the heat generation parameter $S<0$. This is physically true, since increase in thermal conductivity or heat generation enhances the convection current which in return strengthens the boundary layer thickness of the fluid, hence reduces the density of the fluid thereby needs smaller value of Gre to nullify the boundary friction on the plate. However, when boundary plate thickness or heat absorption is increased, the convection current within the channel weakens which raises the required critical values of Gre to nullify the boundary friction. The critical value of Gre increases with increase in viscosity. A general view of this table indicates that, each physical quantity that boosts convection current requires a decrease in Gre while the reverse trend is observed for each physical quantity that 
weakens the convection current.

Table 3 displays the skin friction $\tau$ between the fluid and the plates. It is observed that the skin friction increases at the heated plate with increase in viscosity $\lambda$ while it decreases with an increase in boundary plate thickness. However, the skin friction decreases on the cold plate with increase in viscosity $\lambda$ while a reverse trend is observed when the boundary plate thickness $d$ is increased. On the other hand, when heat generation $S<0$ is increased, then an increase in skin friction is observed on both the plates. It is further observe that the skin friction decreases with increase in heat absorption $S>0$ on both boundary surfaces.

The rate of heat transfer $N u$ is depicted on table 4. It shows that the rate of heat transfer at both the plates increases with increase in thermal conductivity $\varepsilon$. This physically true, since when the thermal conductivity of a fluid is increased, thermal diffusivity becomes high thereby increasing the thermal boundary layer thickness of the fluid, thus increasing the rate of heat transfer. However, the rate of heat transfer decreases with increase in boundary plate thickness $d$ on both the plates. This is due to the fact that the increase in plate thickness reduces the heat flux through the plate to the fluid which in return weakens the convection current of the fluid. It is also observed that the rate of heat transfer decreases on the heated plate with increase in heat generation $S<0$ while a reverse trend is observed on the cold plate. It is further observed that the rate of heat transfer increases on the heated plate with increase in heat absorption $S>0$ but a decrease is observed on the cold plate. This is physically true since fluid temperature decreases with heat absorption thereby reducing the temperature difference between the fluid and the cold plate while it increases same on the fluid interface with the heated plate.

\section{Conclusion}

The present work theoretically investigated the effects of viscous dissipation and boundary plate thickness on a steady mixed convection flow in the presence of variable thermal conductivity and viscosity. The work concluded that the velocity profile increases near the heated plate with increase in $\mathrm{Br}, \mathrm{Gre}$, and $S<0$ while the reverse trends were observed near the cold plate. A decrease in velocity distribution is observed near the heated plate with increase in $\lambda, d, \varepsilon$ and $S>0$ while reverse cases were observed near the cold plate. It is also concluded that the temperature distribution decreases near the heated plate with increase in $B r, \lambda, q$ and $d$ while a reverse cases were observed near the cold plate. It's further concluded that the skin friction and rate of heat transfer decrease with increase in boundary plate thickness $d$. The required critical value of Gre can be obtained by increasing the thermal conductivity while the pressure gradient increases with increase in mass flux. When Brinkman number $(\mathrm{Br})$, viscosity $(\lambda)$, thermal conductivity $(\varepsilon)$, pressure gradient $\left(\frac{d P}{d x}\right)$ and boundary plate thickness $(d)$ are set to zero, the work of Jha and Ajibade [55] is recovered.

\section{References}

[1] Ajay KP, Feffrey RK. Combined forced and natural convection heat transfer in a deep lid-cavity flow. J of Heat and Fluid Flow, 1996;17:460-467.

[2] Jha BK, Deborah D, Ajibade AO. Mixed convection flow in a vertical tube filled with porous material with time-periodic boundary condition: steady-periodic regime. Afr Mat. 2014;26(34):529-543.

[3] Mohamed A. Unsteady mixed convection heat transfer along a vertical stretching surface with variable viscosity and viscous dissipation. J of Egy Math Soc. 2014;22:529-537.

[4] Jha BK, Deborah D, Ajibade AO. Mixed convection in an inclined channel filled with porous material having time-periodic boundary conditions. Trans in Por Med. 2015;2(109):495-512.

[5] Macha M, Naikoti K. Finite element analysis of heat and mass transfer by mhd mixed convection stagnation-point flow of a non-newtonian power-law nanouid towards a stretching surface with radiation. J of Egy Math Soc. 2016;24:458-470.

[6] Jha BK, Aina B. Impact of heat generation/absorption on mhd mixed convection flow in a vertical tube having time periodic boundary condition: steady periodic regime. Heat Pipe Sci and Tech: An intern J. 2016;7:123-147.

[7] Ajibade AO, Onoja UT. Entropy generation and irreversibility analysis due to steady mixed convection ow in a vertical porous channel. Intern J of Heat and Tech. 2017;35(3):433-446.

[8] Jha BK, Aina B. effect of induced magnetic field on mhd mixed convection flow in vertical microchannel. Intern J of Appl Mech and Eng. 2017;22:567-582.

[9] Pantokratoras A. Effects of viscous dissipation in natural convection along a heated vertical plate. Amer J of Comp Math. 2005;29:553-564.

[10] Jha BK, Ajibade AO. Effect of viscous dissipation on natural convection flow between vertical parallel plates with timeperiodic boundary conditions. Comm in Non-Lin Sci and Num Simul. 2012;17:1576-1587.

[11] Kumar PM, Sanchayan M. Viscous dissipation effects on the limiting value of nusselt numbers for a shear driven flow between two asymmetrically heated parallel plates. Front in Heat and Mass Trans. 2012;3:1-6.

[12] Fransisca JS, Tso CP, Dirk R. Heat transfer with viscous dissipation in Couette-poiseuille flow under asymmetric wall heat 
fluxes. Open J of Fluid Dyn. 2012;2:111-119.

[13] Kabir KH, Alim MA, Abdullah LS. Effects of viscous dissipation on mhd natural convection ow along a vertical wavy surface with heat generation. Amer J of Comp Math. 2013;3:91-98.

[14] Mohamed A. Mixed convection flow of a micropolar fluid from an unsteady stretching surface with viscous dissipation. J of Egy Math Soc. 2013;21:385-394.

[15] Prabhakar RB. Effects of thermal diffusion and viscous dissipation on unsteady mhd free convection flow past a vertical porous plate under oscillatory suction velocity with heat sink. Intern J of Appl Mech and Eng. 2014;19:303-320.

[16] RaihanulHaque M, Alam MM, Mohammad M. Effects of viscous dissipation on natural convection flow over a sphere with temperature dependent thermal conductivity in presence of heat generation. Proc Eng. 2015;105:215-224.

[17] Ajibade AO, Umar AM. Steady natural convection Couette flow with wall conduction and thermal boundary condition of third kind. J of Appl Math and Mech ZAMM, 2020;page https://doi.org/10.1002/zamm.201900095.

[18] Michael JS, Dimos P. Effect of microreactor wall conduction on the reforming process of methane. Chem Eng Scie. 2005;6:6983-6997.

[19] Hassab MA, Khamis MM, Shawky IM. The effect of axial wall conduction on heat transfer parameters for a parallel- plate channel having a step change boundary conditions. Num Heat Tran: Part A. 2013;63:430-451.

[20] Ates A, Darici S, Bilir S. Transient conjugated heat transfer in thick walled pipes with uniform heat flux boundary conditions. $5^{\text {th }}$ International conference on heat transfer Fluid Mech and Thermo 2007.

[21] Mei L, Wang QW, Zhixiong G. Investigation on evaluation criteria of axial wall heat conduction under two classical thermal boundary conditions. Appl Ener. 2016;162:1662-1669.

[22] Moukalled F, Darwish M, Acharya S. Influence of wall conduction on mixed convection heat transfer in externally finned pipes. Numer Heat Trans, Part A: Appl. 1995;28(2):157-173.

[23] Kevin DC, Barbaros C. The effects of axial conduction on heat transfer in a liquid microchannel flow. Intern J of Heat and Mass Trans. 2011;54(11-12):2542-2549.

[24] Adrian N, Stefen N, Martins S, Holger V. Interpolation correlation for fluid properties of humid air in the temperature range $100^{\circ} \mathrm{C}$ to $200^{\circ} \mathrm{C}$. Amer Inst of Phy and Amer Chem Soc. 1997;26(4):1111-1123.

[25] Tarakaramu N, Narayana PVS, Venkateswarlu B. Numerical simulation of variable thermal conductivity on 3D flow of nanofluid over a stretching sheet. Nonli Eng. 2020;9:233-243.

[26] Dulal P, Hiranmony M. Effects of temperature-dependent viscosity and variable thermal conductivity on mhd non-Darcy mixed convective diffusion of species over a stretching sheet. J of Egy Math Soc. 2014;22:123-133.

[27] Subhas MAA, Mahesha N. Heat transfer in mhd viscoelastic fluid flow over a stretching sheet with variable thermal conductivity, non-uniform heat source and radiation. Appl Math Mod. 2008;32:1965-1982.

[28] Rahma MM, Mamun AA, Azim MA. Effect of temperature dependent thermal conductivity on magnetohydrodynamics (mhd) free convection flow along a vertical at plate with heat conduction. Nonl Analy: Mod and Contr. 2008;13(4):513-524.

[29] Naseem A.. Visco-elastic boundary layer flow past a stretching plateband heat transfer with variable thermal conductivity.
World J of Mech. 2011;1:15-20.

[30] Vajravelu K, Prasad KV, Chiu-on N. Unsteady convective boundary layer flow of a viscous fluid at a vertical surface with variable fluid properties. Nonl Analy: Real World Appl. 2013;14:455-464.

[31] Singh V, Shweta A. Flow and heat transfer of maxwell fluid with variable viscosity and thermal conductivity over an exponentially stretching sheet. Amer J of Fluid Dyn. 2013;3(4):87-95.

[32] Isaac LA, Anselm 00. Effects of variable viscosity, dufour, soret and thermal conductivity on free convective heat and mass transfer of non-Darcian flow past porous at surface. Amer J of Comp Math 2014;4:357-365.

[33] Hunegnaw D, Naikoti K. Mhd effects on heat transfer over a stretching sheet embedded in porous medium with variable viscosity, viscous dissipation and heat source/sink. Ain Shams Eng J. 2014;5:965-977.

[34] Uwanta IJ, Murtala S. Heat mass transfer ow past an infinite vertical plate with variable thermal conductivity, heat source and chemical reaction. Intern J of Eng and Sci. 2014;3(5):77-89.

[35] Sumayya S, Mohammad RR, Shayan A. Efficiency analysis of straight fin with variable heat transfer coefficient and thermal conductivity. J of Mech Sci and Tech. 2014;26(4):1283-1290.

[36] Devi SPA, Prakash M. Temperature-dependent viscosity and thermal conductivity effects on hydromagnetic flow over a slendering stretching sheet. J of Nig Math Soc. 2015;34:318330.

[37] Animasaun IL. Casson uid ow with variable viscosity and thermal conductivity along exponentially stretching sheet embedded in a thermally stratified medium with exponentially heat generation. J of Heat and Mass tran Res. 2015;2:63-78.

[38] Gopal CH, Jadav K. Effects of variable viscosity and thermal conductivity on magnetohydrodynamics free convection dusty uid along a vertical porous plate with heat generation. Tur J of Phy. 2016;40:52-68.

[39] Jadav K, Hazarika GC. Effects of variable viscosity and thermal conductivity on mhd free convection flow of dusty fluid along a vertical stretching sheet with heat generation. Intern Res J of Eng and Tech. 2016;3(2):1029-1038.

[40] Gopal CH, Bandita P. Effects of variable viscosity and thermal conductivity on magnetohydrodynamics free convection flow of a micropolar fluid past a stretching plate through porous medium with radiation, heat generation and joule dissipation. Tur J of Phy. 2016;40:40-51.

[41] Bandita P. Effects of variable viscosity and thermal conductivity on the unsteady mhd slip flow of micropolar fluid over a vertical plate. Intern J of Comp Appl Tech and Res. 2017;6(7):293-298.

[42] Kareem RA, Salawu SO. Variable viscosity and thermal conductivity effects of soret and dufour on inclined magnetic field in non-Darcy permeable medium with dissipation. Brit J of Math and Comp Sci. 2017;22(3):1-12.

[43] Sreenivasulu P, Poornima T, Bhaskar R. Variable thermal conductivity influence on hydromagnetic flow past a stretching cylinder in a thermally stratified medium with heat source/sink. Fron in Heat and Mass Trans. 2017;9(20):1-7.

[44] Muthtamilselvan M, Gokulavani P, Ramya E, Deog-Hee D. Internal heat generation of dusty uid through porous media over stretching sheet. J of Por Med. 2018;21(9):845-863.

[45] Doh DH, Muthtamilselvan M, Prakash D. Transient heat and mass transfer of micropolar fluid between porous vertical 
channel with boundary conditions of third kind. Intern J of Nonl Sci and Num Simul. 2016;17(5):231-243.

[46] Kairi RR, Ram Reddy C, Raut S. Influence of viscous dissipation and thermo-diffusion on double diffusive convection over a vertical cone in a non-Darcy porous medium saturated by a non-Newtonian fluid with variable heat and mass fluxes. Nonlin Eng. 2017;7(1):65-72.

[47] Kumar A, Alam P, Kapoor S. Numerical investigation of mixed convective flow in parallel channel filled with porous medium. Nonlin Eng. 2014;3(4):203-208.

[48] Rostami AK, Hosseinzadeh K, Ganji DD. Hydrothermal analysis of ethylene glycol nanofluid in a porous enclosure with complex snowake shaped inner wall. Waves in Rand and Comp Med. 2020;1-18.

[49] Hosseinzadeh K, Asadi A, Mogharrebi AR, Ganji MEA. Investigation of mixture fluid suspended by hybrid nanoparticles over vertical cylinder by considering shape factor effect. Journal of Therm Analy and Calo. 2020; https://doi.org/10.1007/s10973-020-09347-x

[50] Mukundan V, Awasthi A. Numerical techniques for unsteady nonlinear burgers equation based on backward differentiation formulas. Nonlin Eng. 2018;7(3):171-181.

[51] Travis JM, Erturk VS. Comparison of the method of variation of parameters to semi-analytical methods for solving nonlinear boundary value problems in engineering. Nonlin Eng. 2020;9:1- 13.

[52] Singh M, Naseem M, Kumar A, Kumar S. Homotopy analysis transform algorithm to solve time-fractional foam drainage equation. Nonlin Eng. 2016;5(3):161-166.

[53] Ajibade AO, Umar AM. Effects of viscous dissipation and wall conduction on steady mixed convection Couette flow of heat generating/absorbing fluid. Intern J of Appl Mech and Eng. 2019;24(4):12-35.

[54] He JH. Homotopy perturbation technique. Comp Meth in Appl Mech and Eng. 1999;178:257-262.

[55] Jha KB, Ajibade OA. Unsteady free convective Couette flow of heat generating/absorbing fluid. Intern J Ener Tech. 2010;2(12):1-9. 